ARTICLE

\title{
Dynamic sex chromosome expression in Drosophila male germ cells
}

\author{
Sharvani Mahadevaraju1,10, Justin M. Fear (10) 1,10, Miriam Akeju, 2,10, Brian J. Galletta ${ }^{3}$, Mara M. L. S. Pinheiro (1D ${ }^{4}$, \\ Camila C. Avelino ${ }^{4}$, Diogo C. Cabral-de-Mello ${ }^{5}$, Katie Conlon², Stafania Dell'Orso (1) ${ }^{6}$, Zelalem Demere ${ }^{2}$, \\ Kush Mansuria ${ }^{2}$, Carolina A. Mendonça ${ }^{4}$, Octavio M. Palacios-Gimenez ${ }^{4,8}$, Eli Ross ${ }^{2}$, Max Savery ${ }^{1}$, Kevin Yu², \\ Harold E. Smith (1) 7, Vittorio Sartorelli [i] 6, Haiwang Yang ${ }^{1,9}$, Nasser M. Rusan ${ }^{3}$, Maria D. Vibranovski ${ }^{4,11}$, \\ Erika Matunis $^{2,11} \&$ Brian Oliver (iD) ${ }^{1,11 凶}$
}

Given their copy number differences and unique modes of inheritance, the evolved gene content and expression of sex chromosomes is unusual. In many organisms the $X$ and $Y$ chromosomes are inactivated in spermatocytes, possibly as a defense mechanism against insertions into unpaired chromatin. In addition to current sex chromosomes, Drosophila has a small gene-poor $\mathrm{X}$-chromosome relic $\left(4^{\text {th }}\right)$ that re-acquired autosomal status. Here we use single cell RNA-Seq on fly larvae to demonstrate that the single $X$ and pair of $4^{\text {th }}$ chromosomes are specifically inactivated in primary spermatocytes, based on measuring all genes or a set of broadly expressed genes in testis we identified. In contrast, genes on the single $Y$ chromosome become maximally active in primary spermatocytes. Reduced $X$ transcript levels are due to failed activation of RNA-Polymerase-II by phosphorylation of Serine 2 and 5.

\footnotetext{
${ }^{1}$ Laboratory of Cellular and Developmental Biology, National Institute of Diabetes and Kidney and Digestive Diseases, National Institutes of Health, Bethesda, MD 20892, USA. ${ }^{2}$ Department of Cell Biology, Johns Hopkins University School of Medicine, 725 N. Wolfe Street, Baltimore, MD 21205, USA. ${ }^{3}$ Cell Biology and Physiology Center, National Heart, Lung and Blood Institute, National Institutes of Health, Bethesda, MD 20892, USA. ${ }^{4}$ Department of Genetics and Evolutionary Biology, Institute of Biosciences, University of São Paulo, SP 05508-090 São Paulo, Brazil. ${ }^{5}$ Instituto de Biociências/IB, Departamento de Biologia Geral e Aplicada, UNESP_Universidade Estadual Paulista, Rio Claro, São Paulo 13506-900, Brazil. ${ }^{6}$ Laboratory of Muscle Stem Cells and Gene Regulation, National Institute of Arthritis and Musculoskeletal and Skin Diseases, National Institutes of Health, Bethesda, MD 20892, USA. ${ }^{7}$ Genomics Core, National Institute of Diabetes and Kidney and Digestive Diseases, National Institutes of Health, Bethesda, MD 20892, USA. ${ }^{8}$ Present address: Department of Evolutionary Biology and Department of Organismal Biology, Systematic Biology, Evolutionary Biology Centre, Uppsala University, 75236 Uppsala, Sweden. ${ }^{9}$ Present address: Department of Pharmacology, Feinberg School of Medicine, Northwestern University, Chicago, IL 60611, USA. ${ }^{10}$ These authors contributed equally: Sharvani Mahadevaraju, Justin M. Fear, Miriam Akeju.. ${ }^{11}$ These authors jointly supervised this work: Maria D. Vibranovski, Erika Matunis, and Brian Oliver. ${ }^{凶}$ email: briano@niddk.nih.gov
} 
O rganisms as diverse as flies and humans have $\mathrm{XY}$ males and XX females. In Drosophila, the $\mathrm{Y}$ chromosome is large, heterochromatin-rich, and gene poor. It carries genes essential for fertility, but not viability ${ }^{1,2}$. The $\mathrm{X}$ chromosome is present as a single copy in males. The small 4 th chromosome is an ancestral $\mathrm{X}$ chromosome $\mathrm{e}^{3,4}$ that is now disomic. Given the sex chromosome copy number differences and their unique modes of inheritance, the unusual evolved gene content and expression of sex chromosomes is widely studied ${ }^{5}$. In many organisms, including mammals ${ }^{6}, C$. elegans ${ }^{7}$, and Drosophila $^{8}, \mathrm{X}$ expression is reduced in testis. Non-mutually exclusive reasons for this reduction include: inactivation of unpaired chromosomes $^{9,10}$, absence of upregulation of the X chromosome in the germline (canonical Drosophila dosage compensation) $)^{11}$, and sexually antagonistic selection followed by evolutionary relocalization of genes required in males from the $\mathrm{X}$ chromosome to the autosomes ${ }^{8,12-14}$. Some combination of these models is possible, if not likely.

Meiotic X-chromosome inactivation is well described in mammals, where premature transcriptional inactivation of the $\mathrm{X}$ and $\mathrm{Y}$ chromosome during mid-spermatogenesis is essential for fertility $^{15}$. The $\mathrm{X}$ and $\mathrm{Y}$ preciously condense into a heterochromatic "XY body" within the pachytene spermatocyte nucleus. Transcriptional inactivation is thought to protect these largely dissimilar chromosomes by defending un-synapsed regions from stealthy invasive transposons that cannot be detected as foreign in the absence of a homolog ${ }^{16}$. Inactivation may also protect against unwanted recombination between the $\mathrm{X}$ and $\mathrm{Y}$, or to repair damage created by lack of recombination ${ }^{17}$. Inactivation might also mark paternal $\mathrm{X}$ chromosomes for later imprinting for inactivation $^{18,19}$. Some of these models are unlikely in the case of Drosophila males, which completely lack meiotic recombination $^{20}$. Exploiting differences, and commonalities, between Drosophila and other models can illuminate causes of unusual sex chromosome expression patterns.

In many organisms, the potentially lethal imbalance of $\mathrm{X}$ linked gene transcripts from the single $\mathrm{X}$ relative to the paired autosomes must be alleviated in the soma to ensure viability ${ }^{21}$. In Drosophila somatic cells, $\mathrm{X}$ chromosome dosage compensation is essential and involves large-scale transcriptional upregulation of the single $\mathrm{X}$ chromosome $\mathrm{e}^{22}$. It is unclear if $\mathrm{X}$ chromosome dosage compensation exists in Drosophila germ cells, which do not require the core dosage compensation machinery used in the soma $^{23,24}$. If dosage compensation occurs, it relies on a undiscovered mechanism. Analysis of transcripts expressed in portions of hand-dissected testes ${ }^{25,26}$ or in mutants that block spermatogenesis in mitotic stages ${ }^{13,21,27}$ suggest that Drosophila testes enriched in spermatogonia show upregulation of the $\mathrm{X}$, consistent with dosage compensation in pre-meiotic cells. Portions of adult testes enriched in late stage spermatocytes ${ }^{25}$ show greatly reduced $\mathrm{X}$ chromosome expression and autosomal genes inserted on the $\mathrm{X}$ show dramatically reduced expression in spermatocytes, far exceeding simple failure of dosage compensation ${ }^{23,24,28}$, consistent with $\mathrm{X}$ inactivation in primary spermatocytes.

Given the dramatic differences in the gonads and gametes between the sexes, the optimal male and the optimal female genome will differ. For autosomes, which reside in each sex in equal dose, selection is balanced. In stark contrast, sex chromosome residency is not balanced. In a population with equal numbers of males and females, $2 / 3$ rds of $X$ chromosomes reside in females. The $\mathrm{X}$ chromosome residency profile is expected to result in more opportunities for selection of alleles favoring females. The Y, of course, resides only in males and is under selection only in males. The presence of a homolog is also important. The single $\mathrm{X}$ and single $\mathrm{Y}$ chromosomes in males are under immediate selection, while only alleles with some degree of dominance are immediately selected in females. Assuming that there is at least subtle dominance ${ }^{29}$, then the $\mathrm{X}$ chromosome should be both feminized and demasculinized (alleles with female advantage selected for, and alleles with male advantage selected against), and the $\mathrm{Y}$ should be both masculinized and defeminized $^{8,13}$. These patterns have been observed in Drosophila species, where expression from the $\mathrm{X}$ chromosome is reduced, and where genes required in males have evolutionarily relocated to other chromosomes ${ }^{12}$. Evolutionary arguments for sex chromosome gene content and expression present an interesting causality dilemma. In the model, antagonistic selection for female functions on the $\mathrm{X}$ drives removal of genes that males need for development from the X. Removal of those genes is permissive for events such as $\mathrm{X}$ inactivation in the male germline $\mathrm{e}^{18}$. It then follows that $\mathrm{X}$ inactivation in the male germline would provide even more selective pressure against $\mathrm{X}$ genes with male-biased functions. In this work, we show that sex chromosome expression is dynamic at tissue and single cell resolutions.

\section{Results}

Sex chromosome expression in different tissues. Drosophila have $\mathrm{X}$ and $\mathrm{Y}$ sex chromosomes, two major autosome pairs and a pair of "dot" 4th chromosomes (Fig. 1a). The Y and 4th chromosomes are gene poor, while the remaining chromosome arms are gene rich (Fig. 1b). To examine sex-biased gene expression patterns, we focused on the distribution of male-biased gene expression across chromosomes or chromosome arms (chromosome elements) for each tissue. We measured adult gene expression (quadruplicates) in the whole body (Fig. 1c) as well as seven tissues (Fig. 1d-j): head, thorax (viscera removed), abdomen (viscera and all reproductive organs removed), viscera (including digestive and excretory organs), reproductive tract (gonads and genitalia removed), terminalia (including genitalia and analia), and gonads in females and males from two strains. We found a significant deviation from random distributions of sex-biased gene expression (we use $p \leq 0.01$ throughout this study) in whole body, head, thorax, viscera, reproductive tract, and gonad ( $\chi^{2}$ test of independence, Supplementary Dataset 1 ). Sex chromosomes and former sex chromosomes are the major contributors to the non-randomness.

Overall gene expression of the sex chromosomes varied by tissue. For X-chromosomes, we observed under-expression of genes in the whole body and gonads of males from either of two wildtype strains (Fig. 1c, j), as previously reported ${ }^{8}$. We also observed reduced X-chromosome expression in heads, thorax, reproductive tract, and terminalia (Fig. 1d, e, h). These nongonadal patterns of $\mathrm{X}$ chromosome expression are difficult to explain by absence of germline $\mathrm{X}$ chromosome dosage compensation or meiotic sex chromosome inactivation, since there are no germ cells in those tissue. By elimination, this suggests that sexual selection drives gene expression patterns of X-chromosome expression in many somatic cell types. Under-representation in gonads could be explained in full or part by the absence of dosage compensation or meiotic sex chromosome inactivation.

Males with no $\mathrm{Y}$ chromosome are viable, but sterile and the $\mathrm{Y}$ chromosome is known to be expressed in spermatocytes ${ }^{30}$. However, the tissue-specific Y chromosome gene expression pattern is poorly described. We report that $\mathrm{Y}$-chromosome gene expression was detectable only in whole males and gonads (Fig. 1c, j).

The $4^{\text {th }}$ chromosome showed a decrease in gene expression in male whole bodies, reproductive tracts, and gonads (Fig. 1c, h, j). As a former $\mathrm{X}$ chromosome, 4 th chromosome expression in the gonads mirrored the $\mathrm{X}$-chromosome underrepresentation of male-biased gene expression. Additionally, and unlike the $\mathrm{X}$ chromosome, 4 th chromosomes are present in two copies in 

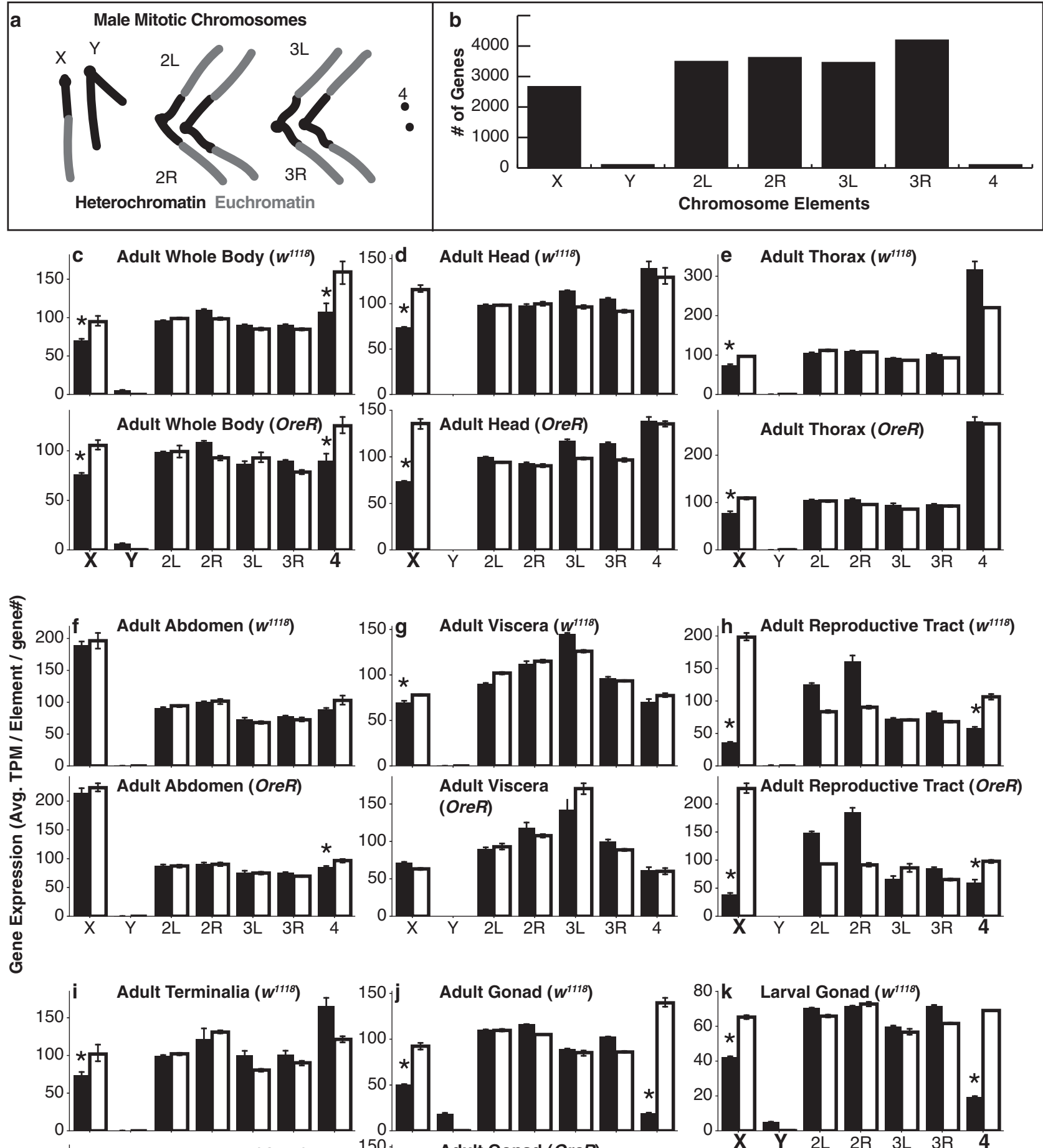

Adult Gonad ( $\left.w^{1118}\right)$

80 k Larval Gonad $\left(w^{1118}\right)$
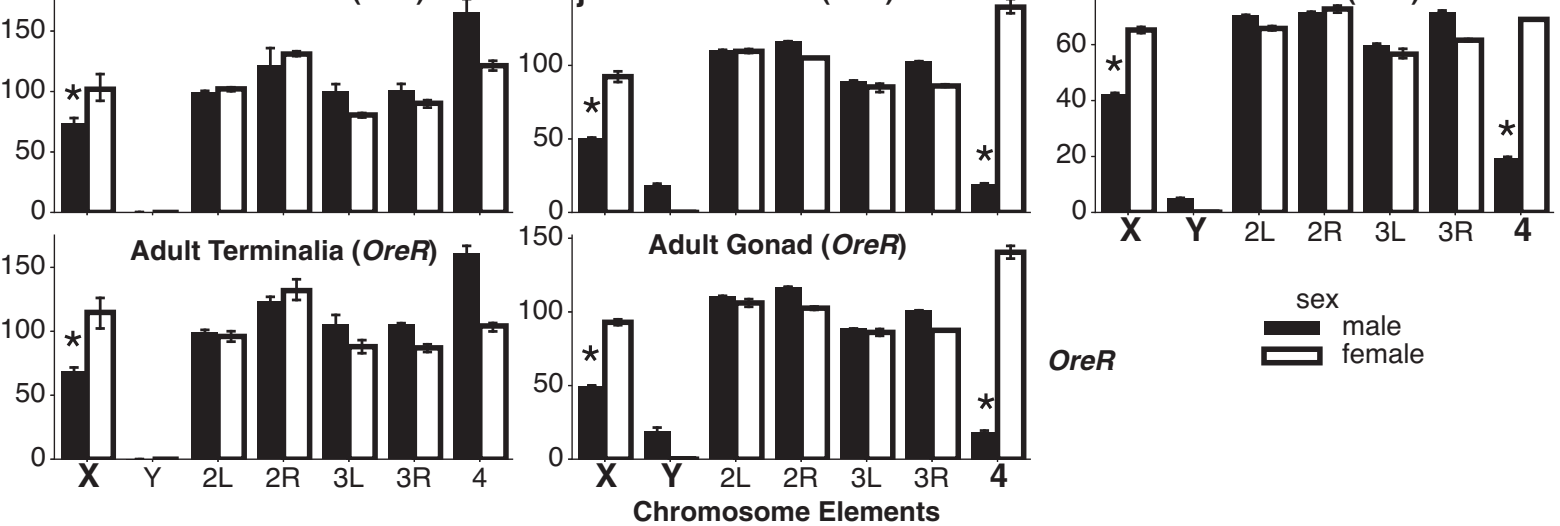

OreR

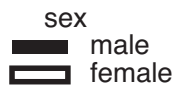

males. Because there are two copies of the 4th chromosome genes, under-representation of expression cannot be explained by the absence of dosage compensation. These data again suggest that there has been sexual selection of sex chromosomes, but only gonads show sex-biased expression of the Drosophila X, Y, and 4 th chromosomes.
Given the dynamic expression of the sex chromosomes by tissue, it is reasonable to assume that there could be differences by cell type and stage within the gonad. Examination of X-linked gene expression from hand-dissected anterior, middle, and posterior regions of adult testes shows a dynamic pattern of $\mathrm{X}$ chromosome expression that decreases as germ cells mature ${ }^{25}$. 
Fig. 1 Bulk RNA-Seq of seven adult tissues and L3 larval gonads. a Illustration of a wild-type male karyotype cartoon depicting the size of the chromosomes and arms (chromosome elements) and the distribution into blocks of heterochromatin (black) and euchromatin (gray). b Haploid annotated gene content of chromosome elements (including non-coding genes) based on FlyBase genome r6.26. c-k For each tissue type, we summed the transcripts per million reads (TPM) of each gene on a chromosome element and divided by the number of genes expressed on that arm. Male (black bars) and female (open bar) gene expression with bootstrapped 95\% confidence intervals of the mean (error bar) are shown. Significant reductions in male relative to female expression (FDR corrected $t$-test for independent samples; female and male at $p \leq 0.01$ ) are shown (asterisk). Where the chromosomal expression showed a sex difference in both strains, the chromosome element is highlighted (bold and in a larger font). Adult tissues: c whole body, $\mathbf{d}$ head, e thorax (viscera removed), $\mathbf{f}$ abdomen (viscera, reproductive organs removed), $\mathbf{g}$ viscera (digestive tract and malphigian tubules), $\mathbf{h}$ reproductive tract (gonads and genitalia removed), i terminalia (genitalia and analia), and $\mathbf{j}$ gonad. For each tissue, we used two "wild-type" strains, $w^{1118}$ and Oregon- $R$ (Ore- $R$ ), which are indicated in each panel. $\mathbf{k}$ Late third instar larval gonads are from $w^{1118}$. Biological samples were prepared in quadruplicate (c-k) for each sex.

We decided to use single cell RNA sequencing (scRNA-seq) ${ }^{31}$ for a higher resolution picture. We did not want to use read depth to sequence transcriptionally inactive secondary spermatocytes and sperm, so we selected Drosophila third instar larval (L3) testis for our experiments. They contain abundant germ cells, including the critical transition from mitotic spermatogonia to meiotic primary spermatocytes $^{32,33}$. Prior to using L3 testes for single cell analysis, we asked whether the patterns of chromosome-specific gene expression known to occur in adult gonads ${ }^{8}$ are also present at this earlier stage of gonadogenesis. We performed RNA-Seq on eight biological replicates each of cleaned 3rd instar larval testes and ovaries (Supplementary Fig. 1) to compare with adult tissues. The overall profiles, including $\mathrm{X}, \mathrm{Y}$ and $4^{\text {th }}$ chromosome expression are similar in both adult and larval gonads (Fig. 1j, k).

Single cell resolution gene expression in the testis. Determining which cells of the testis contribute to unusual patterns of sex chromosome expression is challenging because of the cellular diversity and dynamic nature of gene expression in the gonad. Drosophila spermatogenesis (Fig. 2a) begins at the apical end of the testis ${ }^{34}$ where germline stem cells divide asymmetrically, generating mitotically active differentiating daughters called gonialblasts that produce clusters of interconnected spermatogonia $(\mathrm{G})$. After their fourth mitotic division, spermatogonia quickly enter pre-meiotic S-phase, becoming early primary spermatocytes $\left(\mathrm{E} 1^{\circ}\right)$ that are initially morphologically indistinguishable from spermatogonia ${ }^{35}$. As early primary spermatocytes traverse an extended G2 phase, they undergo a burst of transcriptional activity that is accompanied by a 25 -fold increase in volume ${ }^{33}$, becoming large middle primary spermatocytes $\left(\mathrm{M} 1^{\circ}\right)$ and then slightly smaller late primary spermatocytes $\left(\mathrm{L}^{\circ}\right)$. Fully mature late primary spermatocytes progress through two rapid meiotic divisions, becoming spermatids. Pairs of quiescent somatic cyst cells (C1-C4) envelope each gonialblast and descendants, differentiating alongside the germ cells they support. Cyst cells are a source of intercellular signals and act as a permeability barrier ${ }^{36,37}$. The entire L3 testis is encapsulated by an epithelial monolayer of pigment cell precursors (P), which become adult pigment cells and are required for joining the gonad with the reproductive tract ${ }^{38}$. The basal end of the L3 testis contains terminal epithelial precursor cells $(\mathrm{T})$ which will ultimately contribute to maturation in the final steps of spermiogenesis $^{39}$.

To capture the transcriptional profiles of the cell types in the Drosophila L3 testis, we dissected staged male third instar larvae, enzymatically removing the associated fat body before dissociating 20-40 testes to a single cell suspension for scRNA-seq ("Methods", Supplementary Fig. 1a-d). We piloted dissociation conditions using fly strains expressing GFP in subsets of cells (Supplementary Fig. 1g, h) coupled with dye exclusion (Supplementary Fig. 1i) (70-75\% viability, $n=1000 /$ replicate), ensuring consistent retrieval of single cell suspensions of viable germline and somatic cells. We then performed scRNA-seq on wild-type
L3 testes. We identified 18,965 single cells across three biological replicates based on the intersection of calls from cell ranger count $^{40}$ and DropletUtils emptyDrops ${ }^{41}$ (Supplementary Dataset 2). Potential cell doublets were detected using scrublet ${ }^{42}$ and removed. Based on preliminary cluster analysis using the 2000 most variably expressed genes, we set the perplexity threshold in Seurat ${ }^{43}$ to 0.3 . This yielded ten clusters, each potentially representing a distinct cell type or state with each of three biological replicates contributing to the clusters (Spearman expression rank correlation $\geq 0.93, p<0.01$, Fig. $2 b$ ). While the replicates show the absence of large effects, systematic errors in reporting the biology were still possible. If we captured the majority of the cells and cell types, then we should observe a strong correlation between RNA-seq from the whole organ and the total of all single cells. Indeed, the correlation between replicated bulk RNA-Seq from whole L3 testes and sum of single cells from L3 testes was significant (Fig. 2c; Supplementary Dataset 3), indicating that major cell types are well represented in our scRNA-seq dataset. Gene Set Enrichment Analysis GSEA on the ten clusters indicated that genes with male-biased expression in whole gonads are also enriched in germ cells relative to somatic cells in the L3 gonad (compare red and blue plots, Fig. 2d). Thus, the germline is the major contributor to the pattern of sex-biased expression of the $\mathrm{X}, \mathrm{Y}$, and 4 th chromosomes.

To align gene expression patterns with cell types, we first identified genes with preferential expression in particular cell types, then compared the expression profiles of cells in each of the ten groups with that of all remaining cells in an iterative process for each cell cluster (Fig. 2e, Supplementary Dataset 2). To determine what the cell types were, we projected genes with wellknown expression patterns, carefully re-curated from published images (Supplementary Dataset 4), onto the cell level expression profiles. We also used transgenic protein-trap reporter genes ${ }^{44}$ to identify expression patterns for genes with enriched expression in each cell type (Fig. 2f-k, Supplementary Fig. 3, Supplementary Dataset 4). This allowed us to annotate all ten clusters. $91 \%$ of 79 genes from the literature or reporter expression patterns overlapped cell types identified by scRNA-seq. $46 \%$ were expressed in the same or developmentally preceding cell types, and an additional $45 \%$ were expressed in the same cell lineage. The $7 \%$ of genes showing discordant assay-dependent expression patterns were protein-trap reporters showing either weak or widespread expression, both of which hinder assignment to specific cell types (Supplementary Fig. 3, Supplementary Dataset 4). These data indicate that we have identified many of the major cell types in the L3 testis. We did not identify previously unidentified cell types and failed to unambiguously identify cells in the stem cell niche.

Spermatogonia $(\mathrm{G})$ were characterized by the biased expression of 624 genes (Fig. 2e \#1) including: known early germ cell markers argonaute 3 (AGO3), aubergine (aub), and vasa (vas) ${ }^{45}$, the early differentiation signal bag-of-marbles $(\mathrm{bam})^{35}$, the mitotic cell marker $p 53^{46}$ (Fig. 2f), the chromatin proteins 


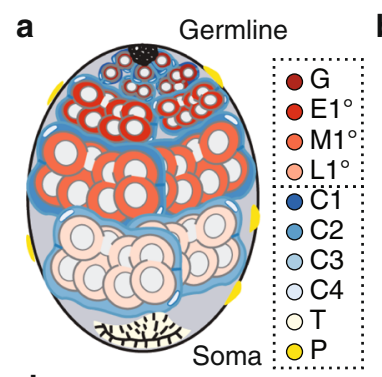

d
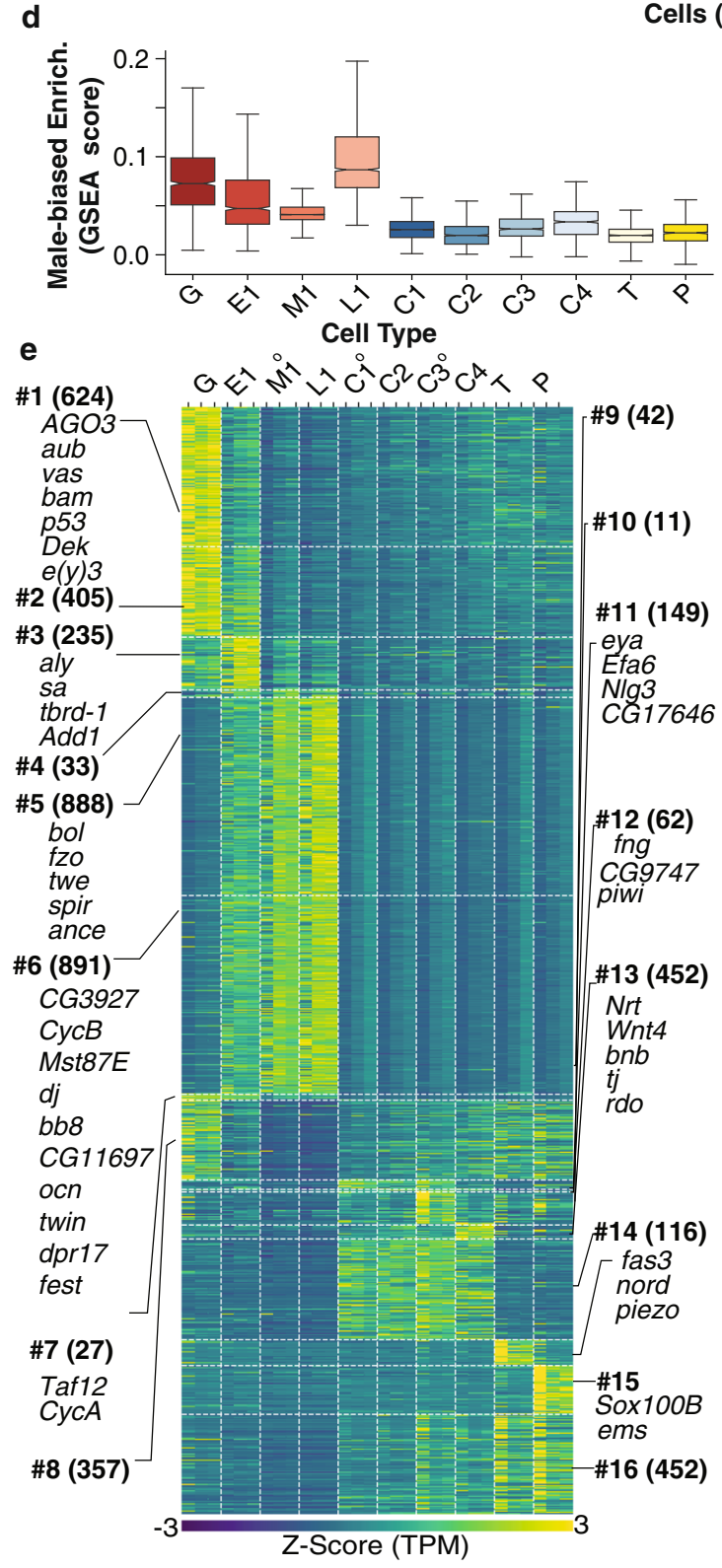

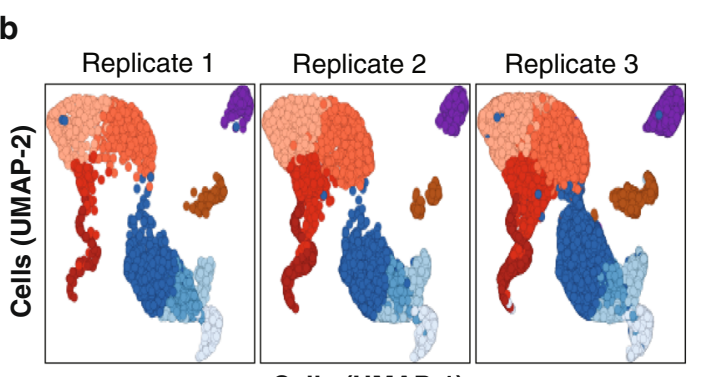

Cells (UMAP-1)
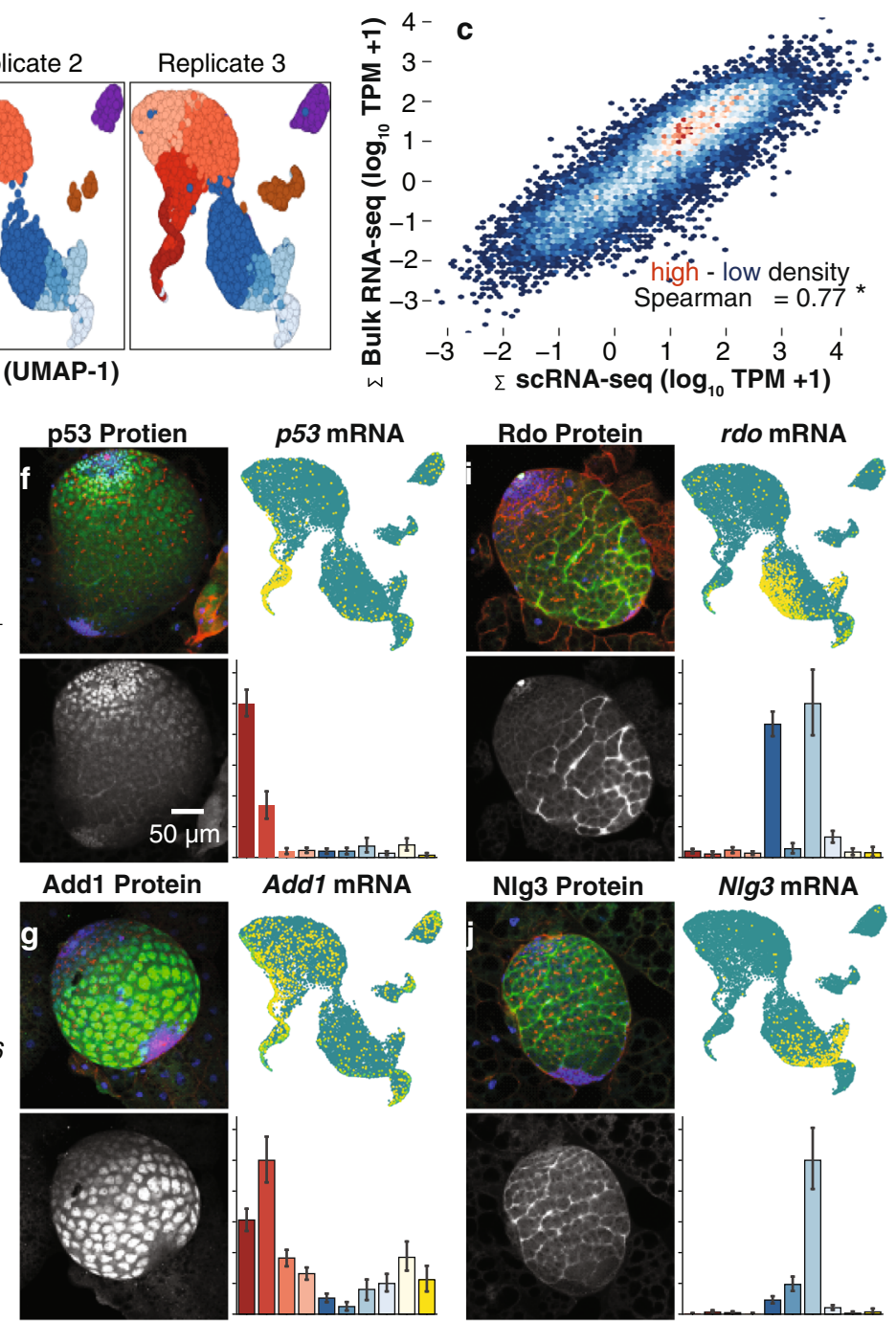

NIg3 mRNA
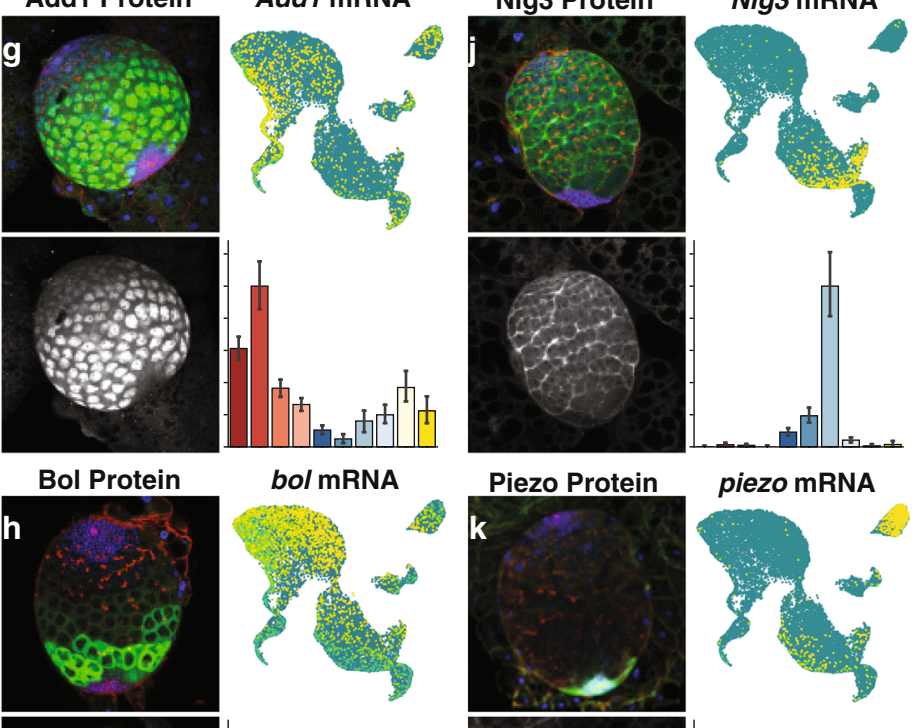

bol mRNA



\section{piezo mRNA}
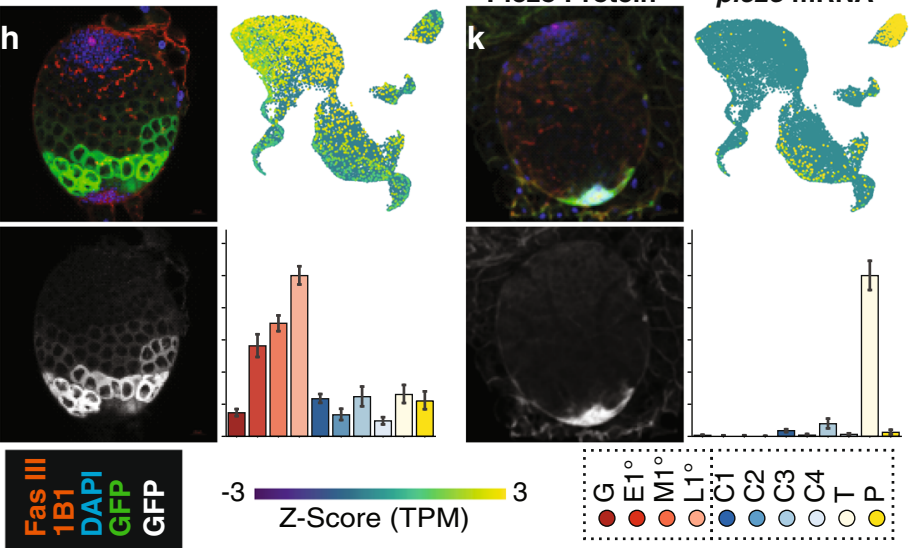

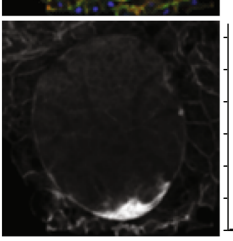

0

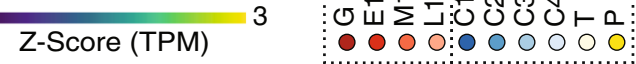

Dek $k^{47}$ and enhancer of yellow $3\left(e(y) 3^{48}\right.$ (Fig. 2e \#1). While expression of these spermatogonial genes also extended to spermatocytes, we could readily distinguish spermatogonia from spermatocytes by their lack of spermatocyte-specific gene expression. The earliest primary spermatocytes $\left(\mathrm{E} 1^{\circ}\right)$ were characterized by high expression of 235 genes including three markers of the transition from spermatogonia to spermatocyte: always early (aly), spermatocyte arrest (sa) ${ }^{49}$, and testis-specifically expressed bromodomain containing protein-1 (tbrd-1) $)^{50}$ (Fig. $2 \mathrm{e}$ $\# 3)$. Sa is a testis-specific TATA-binding protein associated factor
(tTAF), Aly serves as a testis-specific meiotic arrest complex (tMAC) member ${ }^{49}$, and $t b r d-1$ is associated with both transcriptional complexes ${ }^{51}$. In addition, we show that ADD domaincontaining protein 1 (Add1), which encodes a heterochromatin associated protein that interacts with Heterochromatin Protein 1 (HP1) to maintain heterochromatin ${ }^{52,53}$, has enriched expression in early primary spermatocytes in the scRNA-seq data. The Add1 protein accumulated throughout spermatocyte stages, consistent with translational control and/or protein stability over several days of germ cell development (Fig. $2 \mathrm{~g}$ ). The tTAFS and tMACs 
Fig. 2 Identification and annotation of larval testis cell types from scRNA-seq and comparison to bulk testis RNA-Seq. a Illustration of the 3rd instar larval testis, showing germline cell types spermatogonia $(\mathrm{G})$; early- $\left(E 1^{\circ}\right)$, middle- $\left(E 2^{\circ}\right)$, and late- $\left(L 1^{\circ}\right)$ primary spermatocytes; four groups of somatic cysts cells (C1-C4), terminal epithelium precursors $(T)$ and pigment cells $(P)$. These cell types are from germline or soma linages (dashed boxes). See abbreviation and color code key used throughout this manuscript. b Consistency of cell type expression profiles. Uniform Manifold Approximation and Projection (UMAP) for each scRNA-seq replicate. Each cell position in multi-dimensional space projected onto the two-dimensional UMAP space and clustered using k-nearest neighbors. c Correlation between whole testis and single cell profiles. Density scatterplot (high density, red and low density: blue); regression line and Spearman rank correlation, $\rho$ ) of gene expression from the sum of all cells from the larval testis scRNA-seq ( $x$-axis) versus bulk larval testis RNA-Seq ( $y$-axis). Transcripts Per Million reads +1 (TPM +1$)$. d Distribution of genes showing testis-biased expression in bulk RNA-Seq using Gene Set Enrichment Scores ( $y$-axis) among cell types ( $x$-axis) defined by L3 testis scRNA-seq. Each bar is a cell type, so sample size is the number of cells in that cell type: $\mathrm{G}=1065, \mathrm{E} 1=1267, \mathrm{M} 1=4570, \mathrm{~L} 1=2667, \mathrm{C} 1=4064, \mathrm{C} 2=1230, \mathrm{C} 3=910, \mathrm{C} 4=945, \mathrm{~T}=1379, \mathrm{P}=868$. Boxplots (box $=$ interquartile range $(I Q R)$, notch $=95 \% 736$ confidence interval of median, whiskers $= \pm 1.5 \times I Q R)$. e Patterns of differential gene expression among putative cell types. Only differentially expressed genes are shown (two-sided Wilcoxon rank-sum test, FDR corrected $P \leq 0.01$ ). Gene expression is summarized ( $Z$-score; low: blue, high: yellow) for each differentially expressed gene (rows) for all cell-type-by-replicate (columns). Genes are ordered and grouped (horizontal dashed lines) manually into 16 classes (numbered) based on differential gene expression. Each class is annotated with the number of genes (parentheses) and the key genes used to identify the cell types (Supplementary Dataset 2). f-k Immunofluorescence images showing protein expression of representative genes from the protein trap reporters used for cell type annotation. $\mathbf{f} p 53, \mathbf{g}$ Add $\mathbf{h}$ bol $\mathbf{i}$ rdo $\mathbf{j} ~ \mathrm{Nlg} 3$, and $\mathbf{k}$ Piezo. Each panel (counter clockwise from top left) consists of color (Fas III, 1B1: red, DAPI: blue, and GFP: green) and grayscale (GFP) images of protein trap expression, normalized gene expression values (normalized to maximum expression) plotted by cell type (Each bar is a cell type, so sample size is the number of cells in that cell type: $\mathrm{G}=1065$, E1 $=$ 1267, $\mathrm{M} 1=4570, \mathrm{~L} 1=2667, \mathrm{C} 1=4064, \mathrm{C} 2=1230, \mathrm{C} 3=910, \mathrm{C} 4=945, \mathrm{~T}=1379, \mathrm{P}=868$. Error bars are bootstrapped $95 \%$ confidence intervals around the mean), and scRNA-seq gene expression projected onto the UMAP (Z-score; low: blue, high: yellow, see key).

initiate expression of a large battery of genes, many expressed exclusively in primary spermatocytes ${ }^{54}$. As expected, middle and late primary spermatocytes $\left(\mathrm{M}^{0}\right.$ and $\left.\mathrm{L1}^{\circ}\right)$ are characterized by the increased expression of two large groups of 1779 genes that are targets of tTAFs and tMACs (Fig. 2e \#5, \#6), including don juan $(d j)^{55}$. One of these genes was ocnus (ocn). ocn transgenes show very poor expression when inserted on the $\mathrm{X}$ chromosome versus when inserted on autosomes, providing some of the strongest evidence that $\mathrm{X}$ inactivation occurs in Drosophila ${ }^{28}$ (Fig. 2e \#6). As spermatocytes progress through differentiation, we observed a concomitant increase in the expression of the meiotic cell cycle regulator twine (twe) and the translational regulator of Twe encoded by boule (bol) ${ }^{56,57}$ by both scRNA-seq and protein-trap analysis (Fig. 2h). These data defined four distinct stages in male germ cell development in larval testis.

The somatic cell types provide an important baseline for analysis of germline expression and contribute to further understanding of these cell types in the testis (Fig. 2e). For example, traffic jam ( $t j)$ and eyes absent (eya) are expressed in early and late cyst cells respectively in both adult and L3 testis $^{58,59}$, and are most highly expressed in distinct cyst cell clusters in this study. There is also a great deal of biology in the cell expression profiles. For example, the transmembrane protein encoded by defective proboscis extension response 17 (dpr17), a neuronal surface label, is enriched in spermatocytes. Genes encoding the visual system Reduced ocelli (Rdo) protein 60 (Fig. 2i), the exchange factor for the GTPase Arf-6 (Efa6) ${ }^{61}$, and the synaptic adhesion molecule Neuroligin $3(\mathrm{Nlg} 3)^{62}$ (Fig. 2j), show dynamic expression patterns in the cyst cells. The terminal epithelium is poorly studied and we report here multiple RNA and protein-trap markers for this cell type, including the homolog of human Neuron Derived Neurotrophic Factor (Nord), as well as the mechanosensory ion channel subunit Piezo ${ }^{63}$ (Fig. 2k), which acts as a stretch sensor and could help regulate transit of sperm from the testis into the seminal vesicle. Our data (See Supplementary Fig. 2 for UMAP projections for each Drosophila gene), along with a similar dataset from adult testis ${ }^{64}$, should be a resource for those studying testis development and physiology.

Dynamic and cell type specific sex chromosome expression. We looked at the dynamics of sex chromosome gene expression in germ cells in addition to all the other cell types from the single cell dataset (Fig. 3, Supplementary Dataset 5). The somatic cell X chromosome expression relative to autosomes hovered near 1.0 despite the 2-fold dose difference, a pattern consistent with the known canonical $\mathrm{X}$ chromosome dosage compensation mechanism in somatic cells, expected to increase expression from the single $\mathrm{X}^{22}$. This dosage compensation mechanism does not exist in germ cells. Nevertheless, spermatogonia showed similar levels of $\mathrm{X}$ chromosome expression relative to autosomes, providing evidence for non-canonical dosage compensation of the $\mathrm{X}$ chromosome in pre-meiotic germ cells. There was a significant decrease in expression of the X chromosome in early, middle, and late primary spermatocytes $\left(\mathrm{M}^{\circ}\right.$ and $\left.\mathrm{L1}^{\circ}\right)$ relative to either spermatogonia or somatic cells. This decrease in $\mathrm{X}$ expression approached 2-fold which could be due to either a loss of dosage compensation in germ cells as they mature into primary spermatocytes, or to the gain of meiotic X-chromosome inactivation during the transition from mitotic spermatogonia to meiotic spermatocytes. At least some genes on mammalian X chromosomes are also overexpressed prior to inactivation ${ }^{65}$, raising the possibility that this dynamic transition in $\mathrm{X}$ chromosome expression in the male germline is conserved.

Expression of 4th chromosome genes parallels what was seen for the $\mathrm{X}$ (Fig. 3b). The expression ratio of the two 4th chromosomes relative to the two sets of major autosomes hovered near 1 . There was a significant decrease in relative 4 th chromosome expression in middle and late primary spermatocytes $\left(\mathrm{M}^{\circ}\right.$, and $\left.\mathrm{L1}^{\circ}\right)$ compared to expression in either spermatogonia or somatic cells. Since we can rule out failed dosage compensation as a cause of 4 th chromosome decreased expression, there must be a gain of inactivation during the developmental transition from mitotic spermatogonia to meiotic spermatocytes. This X chromosome like behavior may reflect the evolutionary history of the 4 th chromosome; specifically, that the 4 th retained $\mathrm{X}$-inactivation after reacquiring autosomal status.

Finally, the entire pattern of $\mathrm{Y}$ chromosome gene expression is inverted relative to that seen for the $\mathrm{X}$ and 4 th chromosomes (Fig. 3c). We observed poor expression of the $\mathrm{Y}$ in somatic cells and spermatogonia, and increased expression in primary spermatocytes $\left(\mathrm{E} 1^{\circ}, \mathrm{M}^{\circ}\right.$, and $\left.\mathrm{L} 1^{\circ}\right)$, concomitant with decreased expression of the $\mathrm{X}$ and 4 th chromosomes. This occurs from expression of a 42 transcriptionally active Y-linked genes (Supplementary Dataset 2), consistent with the diffuse chromatin and Y-loops originally identified by cytology of primary spermatocytes 66,67 . The decrease in $\mathrm{X}$ and 4 th chromosome 

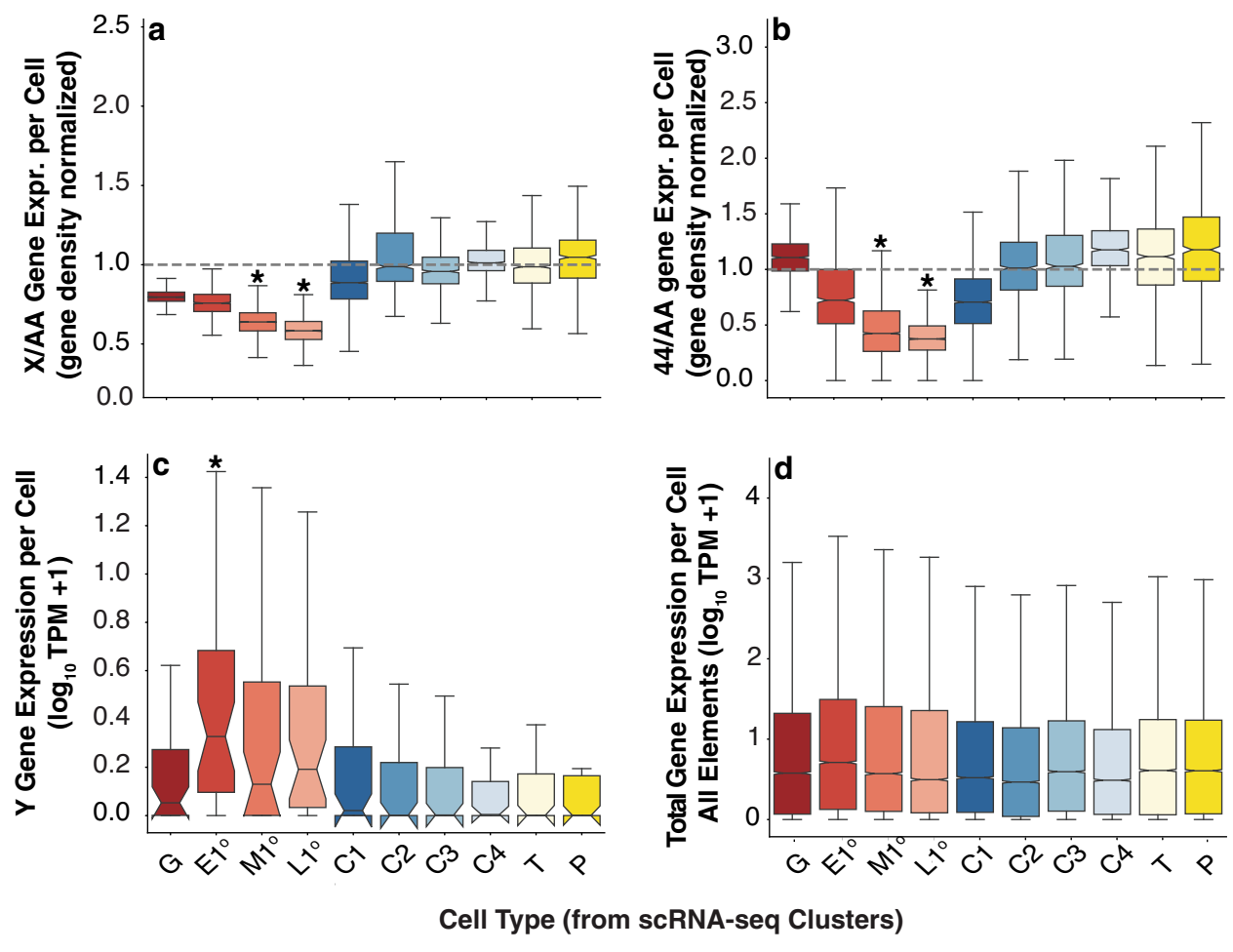

Fig. 3 X-, 4th-, and Y-linked gene expression patterns across cell types. Cell type-specific gene expression of $\mathbf{a} X$-linked genes and $\mathbf{b} 4$ th-linked genes scaled by major autosomal element gene expression (2nd and 3rd chromosomes) at cellular resolution [e.g., (X TPM/ \#X Genes)/(A TPM/ \# A Genes). c $Y$-linked gene expression and $\mathbf{d}$ genome-wide expression in normalized read counts. Chromosomal distributions in germ cells, significantly different from somatic cell types, are indicated with an asterisk ( $\mathbf{a}, \mathbf{b} p \leq 0.01$ using permutation test, $\mathbf{c}: q$-value $\leq 0.01$ using $\chi^{2}$ test). a-d The number of cells scored are: $\mathrm{G}=1065, \mathrm{E} 1=1267, \mathrm{M} 1=4570, \mathrm{~L} 1=2667, \mathrm{C} 1=4064, \mathrm{C} 2=1230, \mathrm{C} 3=910, \mathrm{C} 4=945, \mathrm{~T}=1379, \mathrm{P}=868$. Boxplots (box = interquartile range (IQR), notch $=95 \%$ confidence interval of median, whiskers $= \pm 1.5 \times 1 Q R)$.

expression in middle and late primary spermatocytes does not reflect an overall decrease in total gene expression compared to somatic lineages, as genome-wide expression was robust in all cell types (Fig. 3d). Since the single $X$ and the two 4th chromosomes showed reduced expression, while the single Y showed ongoing expression, these data demonstrate that there is no simple rule for dose-dependent sex chromosome gene expression in Drosophila primary spermatocytes. Genes on chromosomes with no homolog can go up (Y) or down (X), and the 4 th appears to retain X-like reduced expression despite having a homolog. These differences in spermatocyte expression fully account for the differences in X, $\mathrm{Y}$ and 4th chromosome expression observed in bulk RNA-seq of larval testis.

Since genes with high expression in the testis are not uniformly distributed in the genome 8,13 , it was possible that the reduced expression of the $\mathrm{X}$ and 4 th chromosomes was due to the absence of genes highly expressed in spermatocytes rather than a chromosome-wide reduction in expression due to a more global inactivation. A way to avoid this potential confounding effect, is to explore the expression of widely expressed "housekeeping" genes. We explored three data-driven methods to determine $\mathrm{X}$ and 4th chromosome expression of genes with housekeeping functions. In the first two methods, we used low tissue-specificity genes based on Tav and Tissue Specificity Score (TSPS) using our data ${ }^{68,69}$. The third method was a more granular low cell-type specificity metric within in the scRNA-seq experiments (CTSP). Specifically, a set of widely expressed genes expressed in $\geq 33 \%$ of all cells. These methods reduced the expressed gene set numbers to varying degrees, with CTSP being the most stringent (Table 1). The $\mathrm{Y}$ chromosome was expressed in an exquisitely tissue-specific matter and has no widely expressed genes using any metric. To
Table 1 Chromosome element genes and expression.

\begin{tabular}{|c|c|c|c|c|c|}
\hline $\begin{array}{l}\text { Chromosome } \\
\text { element }\end{array}$ & Annotateda & Expressed ${ }^{\mathbf{b}}$ & CTSPc & $\operatorname{T\alpha v}^{d}$ & TSPSe \\
\hline$x$ & 2675 & 2207 & 65 & 336 & 1130 \\
\hline$Y$ & 113 & 42 & 0 & 0 & 0 \\
\hline $2 \mathrm{~L}$ & 3501 & 2767 & 126 & 367 & 1252 \\
\hline $2 \mathrm{R}$ & 3628 & 2861 & 138 & 406 & 1413 \\
\hline $3 \mathrm{~L}$ & 3466 & 2,867 & 101 & 371 & 1347 \\
\hline $3 R$ & 4201 & 3,467 & 145 & 498 & 1673 \\
\hline 4 & 111 & 102 & 3 & 7 & 54 \\
\hline \multicolumn{6}{|c|}{$\begin{array}{l}\text { aGenes annotated in FlyBase r6.26, including non-coding genes. } \\
\text { bGenes detectably expressed in L3 scRNA-seq experiments. } \\
\text { cGenes expressed in } \geq 33 \% \text { of cells in L3 scRNA-seq experiments. } \\
\text { dGenes expressed across multiple adult tissues (tissue specificity score } \tau \leq 0.5 \text { ). } \\
\text { eGenes expressed across multiple adult tissues (tissue specificity score TSPS } \leq 1 \text { ) }\end{array}$} \\
\hline
\end{tabular}

determine if the functions of these three reduced gene sets are consistent with generic gene function, we systematically analyzed Gene ontology (GO) enrichment for all three subsets of genes (Fig. 4a; Supplementary Dataset 5). There are differences in function in the three gene sets. For example, in the Molecular ontology, enzymes were enriched in Tav and CTSP gene sets, while regulators (which are less likely to be generic) were more enriched in the Tav gene set. In the Biological ontology, all three sets were enriched for protein metabolism, consistent with "housekeeping", but the tissue-level Tav and TSPS gene sets were enriched for genes with development and female gamete functions, which is not commonly thought to be generic. Housekeeping genes are often highly expressed. All the reduced gene sets had higher median expression than all expressed genes, 


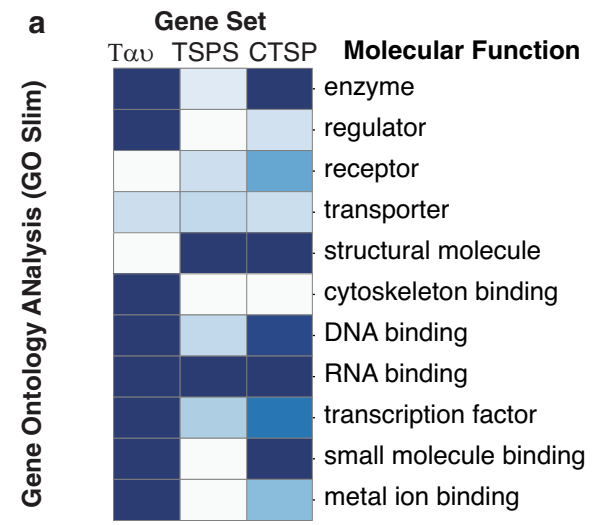

Gene Set
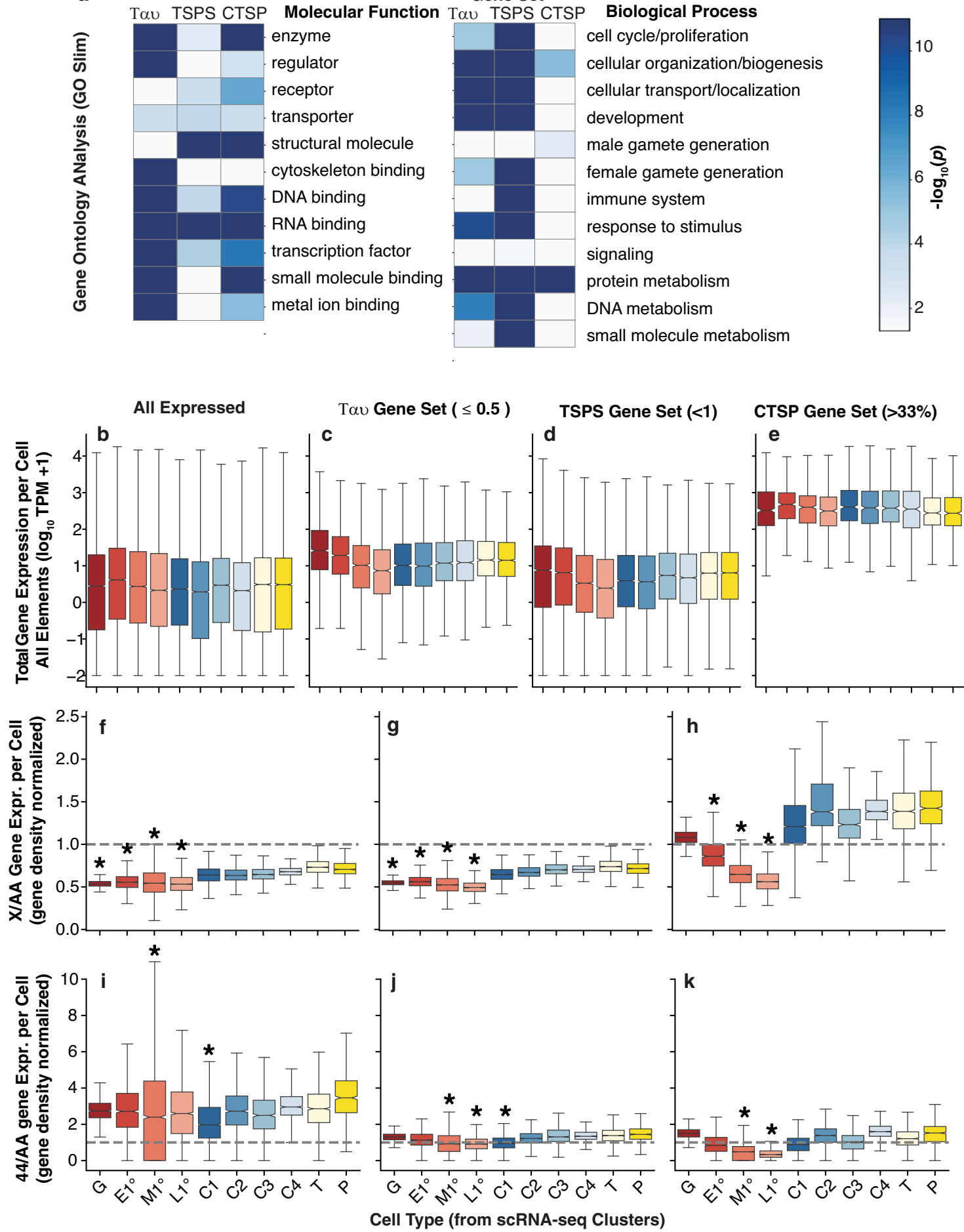

Fig. 4 Gene expression patterns across cell types and chromosome elements for different gene subsets. a Ribbons showing p-values for Gene Ontology enrichments (blue scale) in three gene sets representing widely expressed genes. b-e Total gene expression ( $y$-axis) by cell-type ( $x$-axis) in L3 testis scRNA-seq for different gene subsets. $\mathbf{f}-\mathbf{h}$ X chromosome, and $\mathbf{i}-\mathbf{k}$ 4th chromosome expression relative to major autosomes by cell type as in Fig. 3a, b. $\mathbf{c}, \mathbf{f}$, $\mathbf{i}$ Tau: genes not tissue-specifically expressed ( $\tau \leq 0.5$ from adult tissues Fig. 1). d, $\mathbf{g}$, j TSPS: genes not tissue-specifically expressed (TSPS $\leq 1$ from adult tissues Fig. 1). e, h, $\mathbf{k}$ CTSP: genes not cell type-specially expressed ( $\geq 33 \%$ of cells). The number of cells scored are: $G=1065, E 1=1267, M 1=4570, L 1=$ $2667, C 1=4064, C 2=1230, C 3=910, C 4=945, T=1379, P=868$. Boxplots (box = interquartile range (IQR), notch =95\% confidence interval of median, whiskers $= \pm 1.5 \times \mid \mathrm{QR}$ ). Significance at $p \leq 0.01$ determined by permutation testing (asterisk).

but elevated expression was most pronounced in the CTSP gene set (Fig. 4b-e). Additionally, the CTSP gene set showed greater uniformity in expression levels across cell types. Based on these results, we concluded that the CTSP gene set was the best subset for exploring expression of "housekeeping" genes.
We then used the reduced gene sets to examine the expression of the $\mathrm{X}$ and 4 th chromosomes in all testis cell types. Importantly, when we examined relative expression, all three reduced gene sets showed significantly reduced X/A expression in germline cells (Fig. 4f-h). However, Tav and TSPS gene sets showed reduced X 

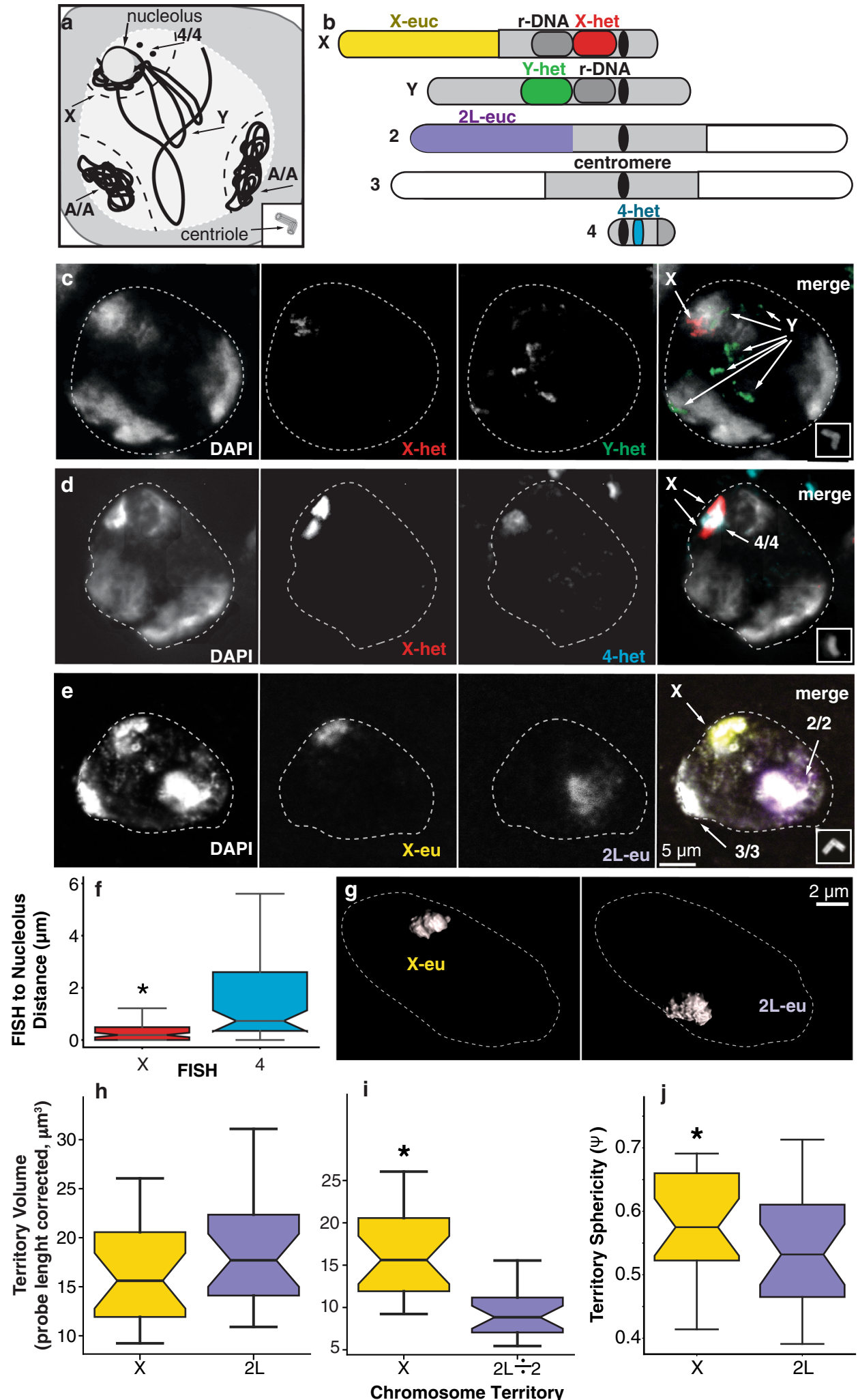

expression in all cell types resulting in $\mathrm{X} / \mathrm{A}$ ratios approaching 0.5 in both somatic and germline cells (Fig. $4 \mathrm{f}, \mathrm{g}$ ). At face value, failed dosage compensation might be expected to approach 0.5 . These observations suggest a reason for the previous conclusion that there is no dosage compensation in male germline ${ }^{11}$ following the analysis of widely expressed genes using tissue-specificity scores. This conclusion is likely spurious, as testis somatic cells express the dosage compensation genes (Supplementary File 1) and the protein complex decorates the $\mathrm{X}$ in those somatic cells as occurs in X-chromosome dosage compensation in other somatic cells ${ }^{23}$. In contrast, the CTSP gene set showed reduced X chromosome expression, approaching 0.5 , only in the late primary spermatocytes (Fig. 4h). Spermatogonia and somatic cells showed X/A rations approaching 1.0. Like the analysis of all expressed genes 
Fig. 5 Spatial location of chromosomal territories in late primary spermatocyte nuclei. a Schematic model summarizing chromosomal spatial location in the spermatocyte nucleus and probe localization on chromosomes. There are two autosomal territories (A/A), $X$ and $4 / 4$ territory close to the nucleolus and $Y$ chromosome. Experimental 1.688 satellite probe to detect $X$ heterochromatin (red), AATAC satellite to detect $Y$ heterochromatin (green), AATAT to detect 4th heterochromatin (cyan), oligo paints to detect $X$-euchromatin (yellow) and $2 \mathrm{~L}$ (purple) are represented on the fly chromosomes (not to scale). b DAPI (gray) distinguishing three chromosomal territories where $X$ and $Y$ satellite probes clearly detects the chromosomes, respectively. Primary spermatocyte nucleus is outlined (white-dashed line) based on DAPI. Asterless was used to stage individual cells by progression of centriole elongation (insets). c, $\mathbf{d}$ Spatial location of $\mathrm{X}$ and 4 th chromosomes. e, $\mathbf{g}$ Representative samples showing spatial location of $\mathrm{X}$ and $2 \mathrm{~L}$ by oligopaints. $\mathbf{f}$ Box plots showing the distributions of mean distances between the $X$ (red), or 4 th chromosomes (cyan), to the nucleolus ( $N=75$ chromosome pairs). To measure chromosome distance to the nucleolus, we averaged the distance from the outer heterochromatin probe edges to the closest nucleolus point ( $p$-value $\leq$ 0.01 two-sided Wilcoxon signed-rank test with continuity correction (asterisk). Images of X and $2 \mathrm{~L}$ spatial localization by oligopaints "masked" in Imaris to obtain 3D images to measure the probe-length corrected $\mathbf{h}$ and the probe-length and copy number corrected $\mathbf{i}$ volume and $\mathbf{j}$ sphericity $(N=23$ nuclei). The sphericity measure accounts for differences in volume: $\phi=\left(\left(\pi^{\wedge}(1 / 3)\right)(6 \text { Volume })^{\wedge}(2 / 3)\right) /($ Area $)$. $\mathbf{h}-\mathbf{j}$ Sample size was 23 . Boxplots (box $=$ interquartile range $(I Q R)$, notch $=95 \%$ confidence interval of median, whiskers $= \pm 1.5 \times I Q R)$. Significance $(p \leq 0.01$, two-sided Welch's $t$-test) (asterisk).

(Fig. 3), the parsimonious explanation is that spermatogonia show dosage compensation and spermatocytes show inactivation or reduced X chromosome compensation.

We similarly examined the expression of the reduced gene sets for the $4^{\text {th }}$ chromosome. Genes with low Tav were overrepresented on the 4 th chromosome, especially in $\mathrm{M}^{\mathrm{o}}$ germ cells and $\mathrm{C} 1$ somatic cells, resulting in an exaggerated overexpression relative to the major autosomes across all cell types (Fig. 4h), while low TSPS and CTSP resulted in significantly lower relative expression of the 4 th chromosomes only in spermatocytes (Fig. 4i, j). The magnitude of spermatocyte decrease was magnified when we used the CTSP gene set, but overall the 4/A ratios were near 1.0 (Fig. 4j). The large sample size of cells resulted in tightly centered distributions, but note that the number of genes contributing the 4 th chromosome measurements was small (Table 1). To briefly summarize, we observed a decrease in $\mathrm{X}$ and 4 th chromosome expression with all genes (Fig. 3) and with reduced gene sets (Fig. 4), suggesting a chromosome-wide change in gene expression in spermatocytes, and not simply a reduced number of X-linked and 4-linked genes with male-biased expression.

$\mathrm{X}$ chromosome condensation in primary spermatocytes. Given that the $\mathrm{X}$ expression falls in maturing primary spermatocytes, we asked if the expression shift in primary spermatocytes correlated with precocious chromosome condensation, as has been previously reported ${ }^{70}$. To locate chromosomes, we used satellite and oligopaint probes that were validated on somatic metaphase chromosomes (Supplementary Fig. 4). The major autosomal bivalents and the X chromosome (along with the 4th chromosomes) reside in three distinct chromosome territories that abut the nuclear envelope of primary spermatocytes ${ }^{71}$ (Fig. 5a). The nuclear interior, more diffusely stained by DAPI, was occupied by the large transcriptionally active $\mathrm{Y}$ chromosome ${ }^{66,67,72}$ (Fig. 5a). The Y chromosome expressed only 42 genes in our experiments, but because of their megabase introns ${ }^{73}$, this represents extensive transcription along the length of the chromosome. In situ hybridization reveals $\mathrm{X}$ chromosome heterochromatic satellite sequences near the prominent spermatocyte nucleolus, where Ribosomal DNA repeats are located, and ribosome biogenesis occurs (Fig. 5b, c, f) ${ }^{74}$. The only homology between the X and Y chromosome is the rDNA clusters. Only the Y-linked rDNAs are expressed; the $\mathrm{X}$-linked cluster is inactive $\mathrm{e}^{75}$, which is consistent with the pattern we observed chromosome-wide. The probes recognizing satellite sequences of the $\mathrm{Y}$ chromosome showed a patchy distribution in the region separating the three main chromosome territories (Fig. 5b, c) ${ }^{66}$, consistent with decondensation and transcriptional activity. The $4^{\text {th }}$ is often, but not always, near the nucleolus ${ }^{72}$. We observed that the $\mathrm{X}$ was universally near the nucleolus (median distance $0.2 \mu \mathrm{m}$ ) and the 4 th was nearly as close (median distance $0.7 \mu \mathrm{m}$ ), well within the same prominent territory (Fig. 5d, f). Since the 4 th occupies the same territory as the $\mathrm{X}$, these chromosomes could be regulated independently, or coordinately, due to territory-level regulation.

The locations of the satellite sequences identify the territories, but we were most interested in chromosome structure in gene rich euchromatic regions. Hence, we used oligopainting to examine the euchromatic portions of the $\mathrm{X}$ chromosome for evidence of compaction that might accompany inactivation. Oligopaint probes show that inactive $\mathrm{X}$ chromosomes have greater compaction (decreased volume) and increased sphericity compared to active $\mathrm{X}$ chromosomes in mammalian cells ${ }^{72,76}$, so we measured both these parameters. We probed similar sized euchromatic regions of the $\mathrm{X}$ chromosome $(22.3 \mathrm{Mb})$ and the left arm of the 2nd chromosome (2 L, 22.7 Mb) with oligopaints (Fig. 5e). We converted raw in situ data in Imaris to create masks $(n=23)$ of pixel intensities (Supplementary Movie 1) and obtained volumetric measurements of the $\mathrm{X}$ and 2 territories (Fig. 5g). We found a that probe length corrected X chromosome volume was reduced relative to chromosome $2 \mathrm{~L}$ (Fig. $5 \mathrm{~h}$ ). This was not significant at the $p \leq 0.01$ level we have used in this work $(p=0.03)$. However, when we corrected the data to account for 2 $\mathrm{L}$ copy number (divided by two), the $\mathrm{X}$ had significantly greater volume than $2 \mathrm{~L}$ (Fig. 5i) which was inconsistent with inactivity resulting from compaction. The assumption that volume scales with copy number is of dubious validity. The $\mathrm{X}$ chromosome was significantly more spherical than $2 \mathrm{~L}$ (Fig. 5j), which was consistent with the hypothesis that compaction accompanies inactivation for regulation of $\mathrm{X}$ expression in Drosophila primary spermatocytes. In mammalian fibroblasts, the inactive $\mathrm{X}$ has a sphericity $(\psi)$ of 0.67 , while the active $X$ has $\psi=0.57^{72,76}$. We found that the Drosophila spermatocyte X had $\psi=0.58$, while $2 \mathrm{~L}$ had $\psi=0.53$. Collectively, these results do not provide strong evidence that Drosophila spermatocyte $\mathrm{X}$ chromosome activity is regulated by overall condensation levels.

Inactive RNA polymerase II mechanism. We more directly addressed transcriptional status of chromosome territories in spermatocytes by determining the phosphorylation status of the regulatory C-Terminal Domain (CTD) of RNA polymerase II (Pol-II). The transcription cycle begins when Pol-II binds the promoter. Serine 5 phosphorylation (pSer5-CTD) causes RNA Pol-II to initiate transcription, and subsequent Serine 2 phosphorylation (pSer2-CTD) induces transcriptional elongation (Fig. 6a) ${ }^{77}$. In the whole testis, we observed pSer2-CTD in spermatogonia and in the two autosomal territories in spermatocytes (Fig. 6b). In spermatocytes, we observed clear pSer2-CTD in two autosomal territories, but the $\mathrm{X}$ and 4 th territory (identified by nucleolar proximity) was poorly stained as previously reported ${ }^{78}$ and pSer5-CTD localization was similar to pSer2-CTD pattern 
a
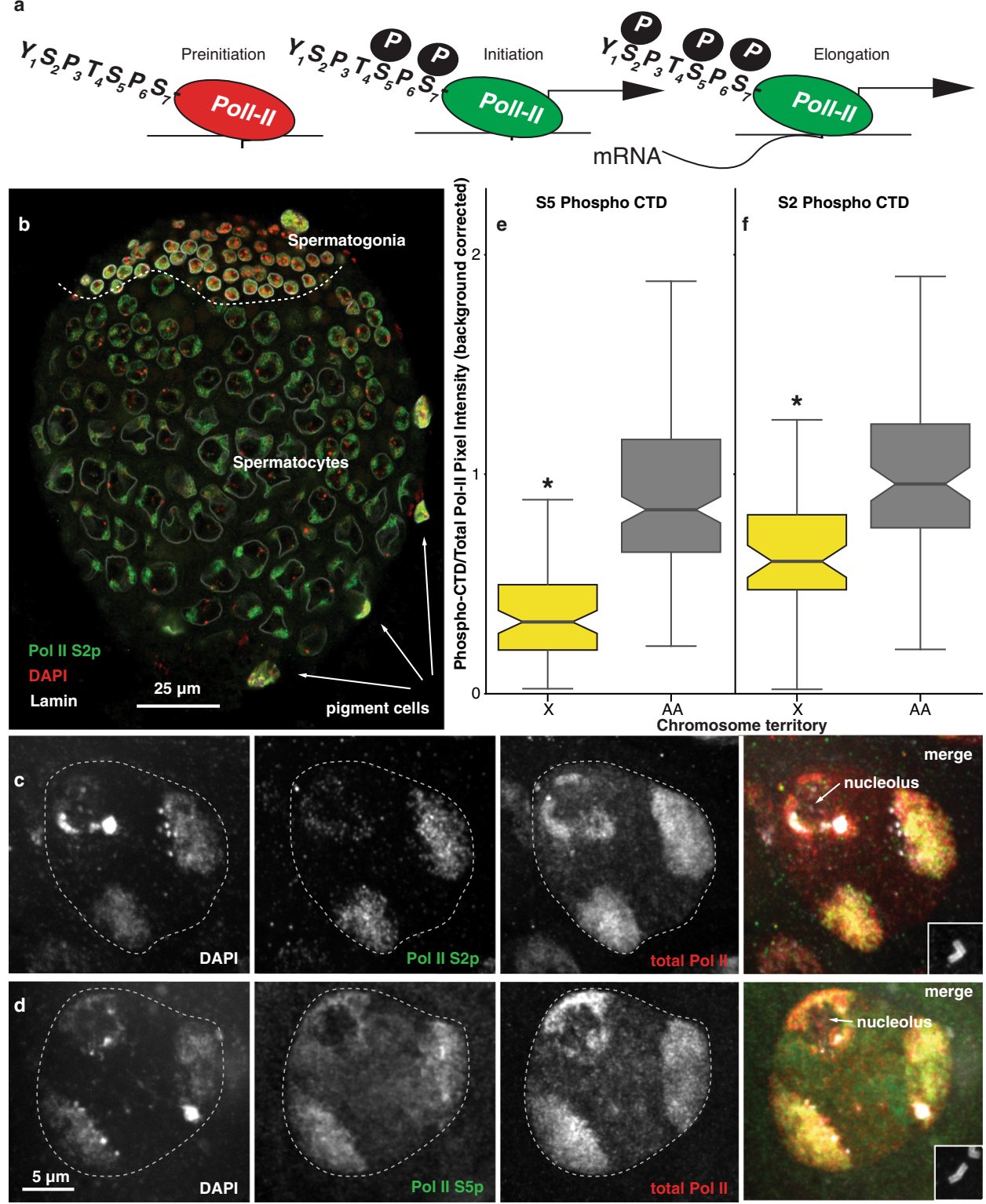

Fig. 6 Transcriptional events in late primary spermatocyte nuclei. a Labelled schematic of RNA Pol-II preinitiation, initiation, and elongation at a promoter. Optical sections of an immuno-stained L3 testis (b) and spermatocytes (c, d). Serine 2 phosphorylated Pol-II localization (green), the nuclear envelope marker Lamin (white), and the DNA stain DAPI (red). We indicate spermatogonia and spermatocytes, separated by a line (dashed, white). Three/five visible pigment cells are indicated. c Serine 2 phosphorylated Pol-II localization (green) and total Pol-II (red). d Serine 5 phosphorylated Pol-II localization (green) with total Pol-II (red). c, d From left to right: DAPI grayscale, Phospho-CTD grayscale, total Pol-II grayscale, color merge. Primary spermatocyte nuclei are outlined (white-dashed line) based on DAPI. Insets in the merged images are anti-Asterless staining (grayscale) used to stage by centriole length, a measure of spermatocyte age. The ratio of e Serine 2 phosphorylated Pol-II (261 territories scored) or $\mathbf{f}$ Serine 5 phosphorylated Pol-II to total Pol-II pixel intensity on the X chromosome and autosome territories ( $N=148$ territories). We corrected for background using non-territory nuclear staining in the same nucleus. Significance ( $p \leq 0.01$, two-sided Welch's $t$-test) (asterisk).

(Fig. 6c, d). We also observe localization of total Pol-II, but very little pSer2-CTD on the $\mathrm{X}$ territory when we co-stained with $\mathrm{X}$ euchromatic oligopaints (Supplementary Fig. 4), consistent with the idea that reduced levels of X chromosome transcripts may be due to decreased active transcription. To obtain more quantitative information on transcriptional status of the $\mathrm{X}$ chromosome, we determined the ratio of pSer2-CTD and pSer5-CTD to total Pol-II within a given territory compared to the autosomes in late primary spermatocytes (Fig. 6c, d). We found a $>2$-fold decrease in pSer2-CTD $\left(\log _{2}=-1.4\right)$ and a $>2$-fold decrease in pSer5-CTD $\left(\log _{2}=-0.6\right)$ on the $\mathrm{X}$ relative to the autosomes in late primary spermatocytes (Fig. 6e, f). These data indicate that the decline in $\mathrm{X}$ chromosome transcripts seen by scRNA-seq is due to a block in the transcriptional cycle regulated by CTD tail phosphorylation. Given that the 4th is also in this territory, it may be subject to the same fate.

\section{Discussion}

Our data clearly support a dynamic model, where X chromosomes are expressed at a higher rate in spermatogonia than one would expect based on DNA copy number alone, supporting the idea of X chromosome dosage compensation in the pre-meiotic male germline. This initial upregulation of $\mathrm{X}$ chromosome expression is followed by a dramatic decrease. We suggest that lower expression of the $\mathrm{X}$ in early meiosis is not due to the 
absence of $\mathrm{X}$-chromosome dosage compensation in the germline ${ }^{11}$, but to an even more extreme reduction in gene expression due to silencing ${ }^{10,79}$. While the canonical dosage compensation pathway, acting to up-regulate $\mathrm{X}$ expression, is absent in male germ cells ${ }^{23,24}$, there is also evidence for non-canonical dosage compensation in testis ${ }^{21,27}$. The mechanism of germline dosage compensation in Drosophila is unknown. Our data provide an important additional argument against failed dosage compensation in the male germline as a cause of reduced $\mathrm{X}$ chromosome expression in spermatocytes, based on the fact that the 4 th chromosome undergoes a similar dramatic decrease in transcript levels, despite being present in two copies. Silencing of genes in spermatocytes is independent of copy number.

Mechanistically, the reduced expression of the $\mathrm{X}$ and 4 th chromosomes in spermatocytes correlates with the failure to activate RNA Pol-II. The Y chromosome is concomitantly active. This begs the question, why? This expression pattern could be due to the simple absence of genes expressed in spermatocytes on the $\mathrm{X}$ and 4 th chromosomes and the presence of genes that must be expressed from the $\mathrm{Y}$ chromosome (Fig. 7a). If there are few genes expressed, there will be little active Pol-II ipso facto. However, expression of "housekeeping" genes suggests a chromosome-wide decrease in $\mathrm{X}$ and 4th expression. A pure gene content model predicts that genes newly arriving on the $\mathrm{X}$ with would be expressed, as evolutionary modification of regulation takes time. In fact, the autosomal ocnus gene is precisely expressed in spermatocytes, but shows extremely reduced reporter activity when inserted onto the $\mathrm{X}^{28}$. This is consistent with a model where the $\mathrm{X}$ is a generally unfavorable environment for spermatocytes gene expression, due to either chromosome- or territory-level repression (Fig. $7 \mathrm{~b}, \mathrm{c}$ ). This is reminiscent of meiotic sex chromosome inactivation in mammals, where $\mathrm{X}$ chromosome expression is high in spermatogonia, followed by $\mathrm{X}$ inactivation associated with a distinct organelle like XY body ${ }^{15}$. The inactivation of both the $\mathrm{X}$ and $\mathrm{Y}$ chromosomes in mammals may be a special case of a more general inactivation of unpaired chromosome regions in a genomic defense model ${ }^{16}$. Lack of homology could signal intruding transposable elements seeking to hijack the germline for vertical transmission to the next generation. Active recognition and silencing would be useful to the host organism. We observed two violations of the prediction that unpaired chromosomes are silenced in primary spermatocytes. Specifically, the 4 th chromosome would be active, and the $\mathrm{Y}$ would be inactive in the simplest versions of this model. However, the 4 th has retained its $\mathrm{X}$ chromosomelike silencing despite having two copies and the $\mathrm{Y}$ is maximally expressed in spermatocytes despite having a single copy. One way to achieve this would be the creation of a repressed territory occupied by both the $\mathrm{X}$ and 4 th chromosomes (Fig. 7c). The evolutionarily retained inactivation of the 4 th could be due to this localization, perhaps originally triggered by monosomy in ancestral species. It is also possible that the non-recombining 4 th chromosome ${ }^{4}$ is not recognized as having a homolog. The single $\mathrm{Y}$ is highly diffuse and very little of it is in this repressed territory. However, allele-specific expression of the Y-linked rRNA genes drive the activity of the nucleolus ${ }^{75}$, so at least part of the $\mathrm{Y}$ is expressed while in a repressed territory. It is possible that Y-linked genes, including the rDNA cluster, required for spermatogenesis escape inactivation as occurs for a subset of $\mathrm{X}$ linked genes on inactive $\mathrm{X}$ chromosomes in mammals ${ }^{80}$. $\mathrm{X}$ to 2 nd or 3 rd chromosome translocations result in breakpointindependent dominant male sterility, whereas $\mathrm{X}$ to 4 th do not $^{74}$. Spreading repression or activation along a chromosome element, or relocation of parts of elements to novel territories might result in such a phenotype. Experiments to test these
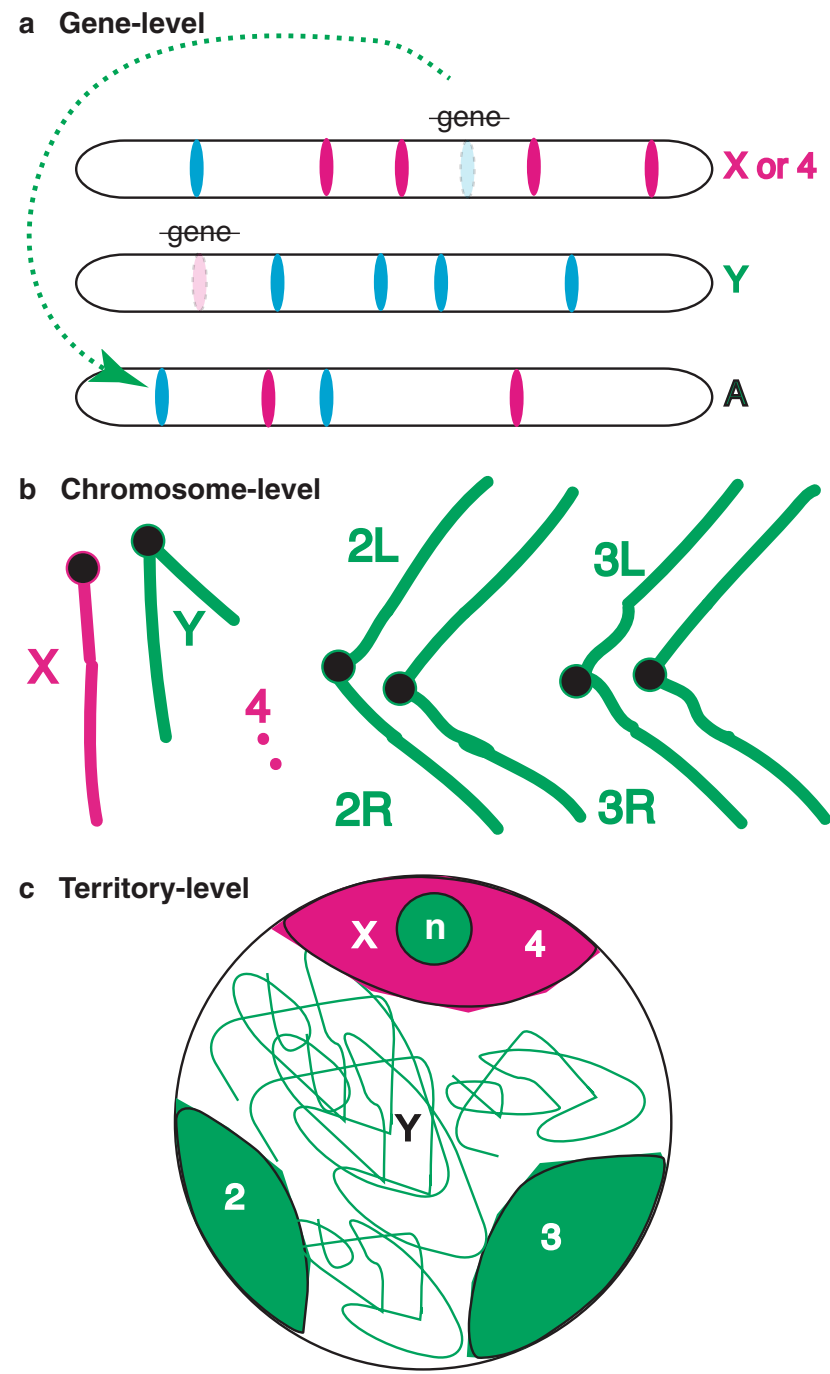

Fig. 7 Models for chromosome element expression in spermatocytes.

Non-mutually exclusive models. a Gene-level regulation. Movement (green dashed arrow) of genes with male-biased expression (blue ovals) off the $X$ due to deleterious changes resulting from antagonistic selection in females or extinction of genes with male-biased expression on the $X$, or femalebiased expression on the $Y$ (strike through) result in a feminized $X$ and a masculinized $Y . X$ and 4th (red), $Y$ (green), and major autosome (black) chromosomes (elongated bars) shown. b Chromosome-level regulation.

This model relies on chromosome-specific regulation, including $X$ chromosome inactivation. Gene expression depends on chromosome elements that are active (green chromosomes) or repressed (red). Centromeres (Black circles). c Territory-level regulation. In this model the $X$ and 4th chromosomes are restricted to a repressed address (red lozenge) within the nucleus (large circle). This could be the result of a barrier preventing activation of Pol-II or active repression within the territory. The 2nd and $3 r^{d}$ chromosome territories (green lozenges) and the diffuse $Y$ (green lines) are expressed normally. The active nucleolus ( $n$, green circle) is embedded within the repressed domain, but depends on expression of $Y$ rDNA, not X rDNA.

models will help us understand the evolution of sex chromosome expression in flies, and probably many other species.

\section{Methods}

Sample preparation. We collected gonads from the immobile third instar (L3) larvae just prior to the final prepupal defecation and prior to eversion of the anterior spiracle (Supplementary Fig. 1a). Staging was aided by staining gut contents using $0.5 \mathrm{mg} / \mathrm{ml}$ Sulforhodamine B yeast paste fed to L3 larvae (clear foregut, 
full midgut/hindgut) (Supplementary Fig. 1b). We dissected L3 larval and 1-5 days old adult gonads and brains directly into phosphate-buffered saline, pH 7.4 (PBS). We used fixed gonads for immunostaining and gonads and brains for in situ hybridization, and gonads for bulk RNA-Seq and scRNA-seq. For expression profiling whole gonads and single cells, we used enzymatic and mechanical treatments to remove non-gonadal tissue. We prepared frozen single-use aliquots of all enzymes used in preparing gonads/cells and tested to determine optimal digestion times. We removed fat body from gonads (Supplementary Fig. 1c-f) using $0.00075 \mathrm{U} / \mu \mathrm{l}$ Papain and $0.0125 \mathrm{U} / \mu \mathrm{l}$ Collagenase in PBS at $22^{\circ} \mathrm{C}$ for $2-3$ $\mathrm{min}$ in $1.5 \mathrm{ml}$ Eppendorf tubes mixed by gently flicking the tube with a forefinger. We decanted floating fatbody cells and washed $2 \times$ with PBS. For whole gonad profiling, we saved the gonads in $350 \mu \mathrm{l}$ RLT buffer mixed two times for $30 \mathrm{~s}$, froze in a dry ice-ethanol bath and stored at $-80^{\circ} \mathrm{C}$. We performed RNA-Seq on eight biological replicates each of cleaned L3 larval testes and ovaries. For single cell preparation, we pre-coated all tubes, pipettes, and filters with $0.5 \%$ Bovine Serum Albumin (BSA) to minimize loss of cells. We removed fat body from gonads as above, except we used $0.075 \%(\mathrm{w} / \mathrm{v})$ porcine powdered pancreas (PPP) rather than papain (Supplementary Fig. 1c, d). We dissociated gonads in $0.45 \%$ PPP and 0.23 $\mathrm{U} / \mu \mathrm{l}$ Collagenase in PBS at $22^{\circ} \mathrm{C}$ for $30 \mathrm{~min}$, teased with tungsten needles and pipetting under the dissecting scope. Digestion was stopped by adding fetal bovine serum (FBS, $1 \%$ final $\mathrm{v} / \mathrm{v}$ ) for $2 \mathrm{~min}$. We decanted the cell suspension and a wash of $0.04 \%$ BSA onto a $35 \mu \mathrm{m}$ cell filter. Cells were pelleted at $845 \times g$ for $5 \mathrm{~min}$ and resuspended in $25 \mu \mathrm{l}$ PBS, $0.04 \%$ BSA. A $1 \mu \mathrm{l}$ cell suspension was used to calculate density microscopically. We performed scRNA-Seq on three biological replicates of L3 instar larval testes.

In initial trials, we determined that the somatic support cells that tightly encase the germline were separated and that the multicellular germline cysts were disrupted using gonads from flies expressing reporters ( $t j>$ GFP or Vasa-GFP) (Supplementary Fig. $1 \mathrm{~g}-\mathrm{h}$ ). The $t j$ gene was highly expressed in a small subset of somatic cyst cells in the testis, these wrap tightly around the germcell clusters, so isolation of single $t j^{+}$cells shows that they were separated from the germ cells (Supplementary Fig. 1g). The vasa gene was expressed in germline cells, which dramatically vary in size (Supplementary Fig. $1 \mathrm{~h}$ ). We also stained cells with $1 \mu \mathrm{g} /$ $\mathrm{ml}$ Hoechst to detect nuclei then examined them microscopically to assess cell morphology. We typically found single cells (96.5\%), but occasionally detected small aggregates of cells $(1 \%$, with $4-8$ cells, each $6-8 \mu \mathrm{m})$, multinucleated cells (2\%, mostly two nuclei spermatocyte cysts) and enucleated cells $(0.5 \%)(n=565$ cells). We analyzed the viability of cells in $0.2 \%$ Trypan blue in $0.5 \times$ PBS, $0.02 \%$ BSA. $25-30 \%$ of cells took up dye, suggesting $70-75 \%$ viable cells prior to microfluidic loading ( $n=1000$ cells) (Supplementary Fig. 1i).

Immunostaining. For immunostaining formaldehyde fixed tissues, we used slightly different conditions depending on the antibodies used and location. For protein traps: we fixed in $4 \%$ formaldehyde in PBS for $20 \mathrm{~min}$; blocked in $1 \times$ PBS plus $0.1 \%$ Triton X-100 (PBX), 3\%BSA, $0.02 \% \mathrm{NaN}_{3}$ and $2 \%$ Normal Goat Serum (NGS) for $30 \mathrm{~min}$; incubated with primary antibodies (1:50 mouse $\alpha$-Fas $3,1: 50$ mouse (1B1) hu-li tai shao, and 1:5000 chicken $a$-GFP in PBX) overnight at $4{ }^{\circ} \mathrm{C}$; washed with 1× PBX; incubated with secondary antibodies (1:200 Alexa Fluor $488 \alpha$-chicken, 1:100 Alexa Fluor $568 \alpha$-mouse) with $1 \mu \mathrm{g} / \mu \mathrm{l}$ DAPI at room temperature for $2 \mathrm{~h}$; rinsed twice in $1 \times$ PBX, once in $1 \times$ PBS; and mounted onto a microscope slide in Vectashield. Images were acquired using Zeiss LSM 800 laser scanning confocal microscopes with $63 \times / 1.4$ Oil DIC M27× objective and illumination lasers at 405 , 488, and $561 \mathrm{~nm}$. For RNA Pol-II: we fixed in 9\% formaldehyde in PBS for $20 \mathrm{mins}$; washed three times in PBS with $0.3 \%$ Triton X-100 (PBST); blocked for $>1 \mathrm{~h}$ in $5 \%$ NGS in PBST at room temperature; incubated in primary antibodies (1:1000 rat a-RNA polymerase II subunit B1, phosphorylated CTD Ser-2, or phosphorylated CTD Ser-5), 1:100 mouse $\alpha$-lamin, 1:30,000 guinea pig $\alpha$-Asl, 1:50 mouse $\alpha$-Fas3, 1:10,000 chicken anti-GFP) in PBX with 5\% NGS overnight at $4{ }^{\circ} \mathrm{C}$; washed $3 \times$ with PBX for $10 \mathrm{~min}$; incubated in secondary antibodies (1:1000 Alexa Fluor 488 goat $\alpha$-rat, Alexa Fluor 568 goat $\alpha$-mouse, and Alexa 647 goat $\alpha$-guinea pig) at room temperature; counterstained with DAPI at 1:1000 in 5\% NGS in PBX for 2-8 $\mathrm{h}$ at room temperature; $3 \times$ in PBX for $10 \mathrm{~min}$ at room temperature and mounted in Aquapolymount under a No. 1.5 coverslip. Images of larval testes were acquired using Nikon Eclipse Ti with a 100×/1.4 NA oil immersion objective, a spinning disc confocal head, a CoolSNAP HQ2 camera and illumination lasers at 405, 491, 561, and $642 \mathrm{~nm}$. The microscope was controlled by and images (Supplementary Fig. 2) were acquired using MetaMorph. All data analysis was performed using ImageJ.

In situ hybridization. To detect heterochromatin on $\mathrm{X}$, 4th and $\mathrm{Y}$ chromosomes, we synthesized probes (1.688 satellite of X chromosome and AATAT repeats for 4 th and AATAC repeats for Y chromosomes) using D. melanogaster genomic DNA as a template 72,81 . PCR was performed using $50-100 \mathrm{ng} / \mu \mathrm{l}$ of template DNA with Taq Platinum DNA polymerase following the manufacturer's instructions. Probes were PCR-labeled with digoxigenin-11-dUTP or biotin-14-dUTP during their synthesis at the $5^{\prime}$ end. To detect the euchromatin, we synthesized oligopaint probes using 5' fluorophore labeled and 5' phosphorylated PCR primers, amplified with the following cycles: $95^{\circ} \mathrm{C}$ for $5 \mathrm{~min} ; 40$ cycles of $95^{\circ} \mathrm{C}$ for $30 \mathrm{~s}, 53^{\circ} \mathrm{C}$ for $30 \mathrm{~s}$ and $72{ }^{\circ} \mathrm{C}$ for $15 \mathrm{~s}$, with a final extension step at $72^{\circ} \mathrm{C}$ for $5 \mathrm{~min}$. The PCR product was purified using Zymo spin columns (D4031) and then digested using lambda exonuclease for $30 \mathrm{~min}$ at $37^{\circ} \mathrm{C}$ and $10 \mathrm{~min}$ at $75^{\circ} \mathrm{C}$. The digested probe products were precipitated using ethanol and quantified using a nanodrop.

We stained mitotic chromosomes to assess probe hybridization (Supplementary Fig. 3). For mitotic metaphase chromosomes, we immersed dissected brains in 0.05\% Colchicine in PBS for $20 \mathrm{~min}$, hypotonized in tap water for $15 \mathrm{~min}$, and fixed in 3:1 (v/v) ethanol:acetic acid for $15 \mathrm{~min}$, transferred to $60 \%$ acetic acid and squashed on a slide. Fluorescence in situ hybridization for heterochromatic probes was performed according to Pinkel et al. ${ }^{82}$ with modifications ${ }^{83}$ using mitotic chromosome spreading or spermatocytes from adult testes. Post-hybridization washes were performed as follows: two times in $2 \times \mathrm{SSC}$ at $42^{\circ} \mathrm{C}$ for $5 \mathrm{~min}$, two times in $0.1 \times$ SSC at $42^{\circ} \mathrm{C}$ for $5 \mathrm{~min}$, one time in $2 \times$ SCC at $42^{\circ} \mathrm{C}$ for $5 \mathrm{~min}$ and finally in $2 \times$ SSC at room temperature for $10 \mathrm{~min}$. Probes labeled with biotin-16dUTP were detected using avidin-FITC conjugate (Fisher Scientific) and probes labeled with digoxigenin-11-dUTP were detected using anti-digoxigenin rhodamine (Roche). The preparations were counterstained using DAPI and mounted in Vectashield. Images from metaphase spreads and adult spermatocytes were obtained using a Zeiss Axiophot 2 microscope equipped with Bright field and epifluorescence optics.

For fluorescence in situ hybridization using oligopaints, we fixed testes in 5\% formaldehyde washed in 1X PBX and then $0.3 \mathrm{M} \mathrm{NaCl}, 30 \mathrm{mM} \mathrm{Na}$ Citrate, $1 \%$ Tween $20(2 \times$ SSCT $)$, then successively in 20,40 , and $50 \%$ formamide in $2 \mathrm{X}$ SSCT. Testes were pre-denatured at $37^{\circ} \mathrm{C}$ for $4 \mathrm{~h}, 92^{\circ} \mathrm{C}$ for $3 \mathrm{~min}$, and $60^{\circ} \mathrm{C}$ for $20 \mathrm{~min}$ in $50 \%$ formamide in $2 \times$ SSCT. We heated $100 \mathrm{pmol}$ of the X-euchromatin primary probe and $200 \mathrm{pmol}$ of the $2 \mathrm{~L}$-euchromatin primary probe in probe buffer with RNase A and testes to $91^{\circ} \mathrm{C}$ for $3 \mathrm{~min}$ and incubated overnight at $37^{\circ} \mathrm{C}$ for $18 \mathrm{~h}$ on a rocker. We washed testes in $50 \%$ formamide $+2 \times$ SSCT. Testes were then washed with decreasing concentrations of formamide (50 and 20\%) and $2 \times$ SSCT. To counterstain with antibodies, we refixed with $16 \%$ Formaldehyde, 100\% Tergitol, 10× PBS, heptane and MilliQ H20, washed in $1 \times$ PBX, and then blocked in a $1 \times \mathrm{PBX}+1.5 \% \mathrm{BSA}$ mixture for $1 \mathrm{~h}$. Testes were incubated overnight at $4^{\circ} \mathrm{C}$ with: 1:20 mouse $\alpha$-lamin, 1:20 mouse $\alpha$-lamin $\mathrm{DmO}$, and 1:10,000 guinea pig $\alpha$-Asl in PBX. Testes were washed in $1 \times$ PBX and then incubated in the secondary antibodies. Testes were mounted onto a microscope slide containing a drop of Vectashield. Images were acquired using Zeiss LSM 800 laser scanning confocal microscope with $63 \mathrm{X} / 1.4 \mathrm{NA}$ oil immersion objective and illumination lasers at $405,488,561$, and $640 \mathrm{~nm}$.

Image analysis. To measure the $\mathrm{X}$ and $2 \mathrm{~L}$ oligopaint territories, we loaded CZI images into Imaris. Centriole elongation is a marker of time during the prolonged G2 phase of primary spermatocyte development, which we scored using the Asterless (Asl) centriole marker. We created X and $2 \mathrm{~L}$ oligopaint probe channel masks ( $n=23$ for both $\mathrm{X}$ and $2 \mathrm{~L}$ ) of the pixel intensities in late primary spermatocyte cells. We measured volume and sphericity (shape) of the masks and performed Paired $t$-tests.

For quantitative measurements of Ser-2, Ser-5 Pol-II, and total Pol-II signal, samples were dissected and processed in parallel for each experiment and imaged (at 16 bits) on a single day using identical microscope settings ensuring that all pixel intensities were within the dynamic range of the camera (no more than $3 / 4$ of the full dynamic range). We normalized $\mathrm{X}$ chromosome territory region of interest to the average total fluorescence intensity of the autosomes for each measurement in late apolar spermatocytes determined from that day's experiment. The data presented are from two independent experiments. To quantify the amount of phosphorylated CTD Ser-2 or Ser-5, total Pol-II and DAPI in the discrete DNA domains within the nuclei of spermatocytes, autosomal or nucleolar associated DNA domains that were spatially separated from other domains (in XY and with no overlap in the Z-slices containing any signal from the cluster) were selected from images of spermatocytes. Z-slices encompassing the domain were then summed and the integrated pixel intensity for this volume was measured. An area of nucleoplasm not containing any other DNA domains was selected and measured for background subtraction. X chromosomal domain was identified by the nucleolus region and the other two were considered as autosomal domains. Ratios between X chromosomal and summed autosomal domains were calculated. We costained with antibodies to Pol-II and FISH oligopaints to confirm localization patterns to $\mathrm{X}$ and 2 territories (Supplementary Fig. 4).

RNA-Seq-library preparation. For bulk sequencing, we added fatbody-free gonads to RNeasy 96 kit $350 \mu$ RLT buffer mixed two times for 30 s, froze in a dry ice-ethanol bath and stored at $-80^{\circ} \mathrm{C}$. We extracted total RNA from gonad samples using RNeasy 96 kit following the manufacturer's protocol. We quantified RNA using the Quant-iT RiboGreen quantification kit and used $200 \mathrm{ng}$ of total RNA for library preparation ${ }^{84}$. We spiked in External RNA Control Consortium (ERCC) spike-ins pools $78 \mathrm{~A}$ and $78 \mathrm{~B}$ (transcribed from a certified standard reference) prior to fragmentation. For multiplexing, we used eight different TruSeq v2 kit barcoded adaptors. We sequenced stranded multiplexed libraries using a single-ended $50 \mathrm{bp}$ strategy and generated RNA-Seq profiles of biological quadruplicates on a HiSeq Illumina 2500.

For single-cell sequencing, we loaded dissociated cells (6 K for Replicates 1 and $2,12 \mathrm{~K}$ for replicate 3 ) onto the 10X Chromium system for barcoding and library preparation following the user guide for Single Cell $3^{\prime}$ Reagent Kits v2. We quantified libraries with Quant-iT PicoGreen and confirmed 300-500 bp insert 
sizes on a TapeStation 2200. We generated scRNA-Seq profiles in biological triplicate pools on the $10 \mathrm{X}$ Chromium System and sequenced $(\operatorname{Read} 1=26 \mathrm{bp}$ Read $2=98$ bp and Readi7 $=8$ bp) on a HiSeq Illumina 2500 .

RNA-Seq-data processing. For the bulk RNA-Seq, we demultiplexed and converted Binary Base Call (BCL) files to FASTQ using bcl2fastq (v2.17.1.14, Illumina, San Diego, CA, USA). We processed FASTQ files through custom RNA-Seq workflow (commit: 0609e5c8752, Supplementary Dataset 6). Briefly, reads are trimmed of Illumina adapters using cutadapt ${ }^{85}$ with default parameter except for --q 20 and --minimum-length $=25$. We mapped with Hisat $2^{86}$ with default parameters except --max-intronlen 300,000 and --known-splicesite-infile using annotated splice sites (FlyBase r6-26). We removed multi-mapping reads and lowquality alignments using samtools view -q $20^{87}$. Gene expression and intergenic expression (FlyBase r6-26) is quantified in a strand-specific manner using Fea tureCount from the subread package ${ }^{88}$ with the $-\mathrm{s} 2$ option. The workflow outputs a number of quality control metrics from FastQC, FastQ Screen ${ }^{89}$, Picard MarkDuplicates, and Picard CollectRnaSeqMetrics (Supplementary Dataset 6). In addition, we quantified intergenic expression and ERCC spike-in expression using FeatureCount ${ }^{90}$. See Supplemental Dataset 1 for visualization of all analyzed scRNA-Seq data and all code is available at the Zendo repository: https://doi.org/ 10.5281/zenodo.3973174. Bulk RNA-Seq data is available at the Gene Expression Omnibus (GEO) GSE115478 (larval testes) and GSE115511 (larval ovaries). Bulk adult testes and ovaries are from GSE99574 ${ }^{91}$, Supplementary Dataset 3.

For scRNA-Seq, we converted BCL files to FASTQ using cellranger mkfastq (https://support.10xgenomics.com/single-cell-gene-expression/software/pipelines/ latest/what-is-cell-ranger, Supplemental datset 6). We demultiplexed cells, aligned reads, and quantified gene-level expression using cellranger count with default parameters. For alignment and quantification, we used the D. melanogaster genome assembly (dm6) with gene annotations from FlyBase r6.26. Larval testes scRNASeq data is available at the GEO GSE125947.

To quantify how well scRNA-Seq captured the overall expression patterns of the testis, we summed gene-level counts from all cells for each of our three replicates and normalized scRNA-Seq and bulk RNA-Seq libraries using Transcripts Per Million reads $+1(\mathrm{TPM}+1)$. We calculated pairwise Spearman rank correlation coefficients among all samples (Supplementary Dataset 3).

Sex-biased gene expression. For sex-biased gene expression, we used publicly available adult RNA-Seq counts from for $w^{1118}$ and Oregon-R (GEO: GSE99574). Briefly, counts tables from $w^{1118}$ and Oregon- $R$ were downloaded for each tissue. Gene identifiers were mapped to current FlyBase FBgns using the provided annotation files and FlyBase's secondary ID. We estimated differential gene expression by comparing males and females using DESeq2 for each adult tissue and for L3 bulk RNA-Seq generated in this study.

scRNA-seq cell selection, clustering, and cell type annotation. Prior to the downstream analysis, we removed cell IDs that were likely to be empty and identified cell IDs that likely contain two or more cells known as multiplets (Supplementary Dataset 2). There are two distinct classes of multiplets: homotypic and heterotypic. Homotypic multiplets contain 2 or more cells from the same celltype. Homotypic multiplets tend to have high UMI counts and are found at the top of the UMI distribution however, setting an upper UMI threshold may bias against high RNA content cell-types. To identify an optimized upper UMI threshold, we performed a grid search over $[4000,5000,6000]$ gene expression thresholds. For each point in the grid search, we clustered cells using Seurat ${ }^{43}$. We then compared cluster calls using the adjusted rand index and selected an upper UMI threshold which did not drastically change clustering. We found that cluster calls remained stable using an upper threshold of $\leq 5000$ expressed genes, which removed 768 cells that were putative homotypic doublets. Heterotypic multiplets contain 2 or more cells from different cell-types. This leads to a mixture of expression profiles causing cells to look like intermediate cell-types, which are identifiable using in silico mixing of cell-types. We removed 410 cell IDs that behave like the mixture.

We combined all three scRNA-Seq replicates into one dataset using single-cell integration ${ }^{92}$ and clustered cells with K-Nearest Neighbors using the 2,000 most variably expressed genes ${ }^{43}$. To determine cluster boundaries, we iteratively tested multiple threshold parameters (range $0.2-1.2$ ), selecting a threshold of 0.3 , which gives a set of 10 clusters that clearly separate germline and somatic cyst lineages, and each cluster is represented in all three replicates (Supplementary Dataset 2).

We compared gene expression patterns between scRNA-Seq and manually curated images (43 genes from the literature; 31 genes from this study; Supplementary Dataset 4 ). We developed an overlap score (0-4) between scRNASeq expression and mRNA or protein expression in curated images. A score of 4 indicates a gene showed cell type biased expression (scRNA-Seq) in the exact same cell types as mRNA or protein expression (curated images). Since protein expression may lag transcription, we also gave a 4 when protein expression was later in the specific cell lineage. A score of 3 indicates that the gene is highly expressed, but not cell type biased, in the exact same cell types as mRNA or protein expression. A score of 2 indicates cell type biased gene expression in the same cell lineage as mRNA or protein expression. A score of 1 indicates high gene expression in the same cell lineage as mRNA or protein expression. Finally, a score of 0 indicates that there is no overlap.

Projecting gene expression onto the UMAP clusters is a visually appealing method to rapidly access where genes are expressed in the testis. We generated these projections of cellular resolution expression for every expressed gene in the Drosophila genome (FigShare https://doi.org/10.35092/yhjc.11950746). For each gene we plot a UMAP project colored by $Z$-score value $(-3,3)$. The line graph represents normalized gene expression for each cluster.

ScRNA-seq downstream analysis. We know that the transcriptional profile of the testis is unique and expressed genes are not equally distributed among chromosome elements. Therefore, we wanted to interrogate a multiple gene sets to ensure our observations are unbiased. We created three "housekeeping" genes sets, two based tissue-specific expression in adult tissues and two gene sets based on scRNA-seq gene expression from L3 testis. Using the adult tissue panel described in "Sex-biased gene expression", we compared gene counts across all adult tissues using two tissuespecificity estimates Tau and TSPS. We consider genes with $\tau \leq 0.5$ or TSPS $\leq 1$ as not tissue specific (i.e., "housekeeping"). We consider genes expressed anywhere in our scRNA-Seq data (expressed) or gene expressed in at least 33\% of cells (low cell type specificity, CTSP) which are effectively genes expressed across multiple cell types.

Expression by chromosome arms is complicated as gene expression of an individual cell is sparse in scRNA-Seq, and simple aggregation of cell counts is not appropriate because missingness is not random ${ }^{93}$. Therefore, we used a cell level permutation test approach for all downstream analysis. Briefly, we calculate each the measure of interest (see below) for each individual cell using all gene sets (see above). For pairwise comparisons, we compare the distributions of cells from two cell types using Mann-Whitney $U$ test. We then permute cell type labels and repeat the Mann-Whitney $U$ test comparisons for 10,000 iterations. We calculate the overall $p$-value based on the number of times the permuted Mann-Whitney $U$ test was more extreme than the original observed test. For mean comparisons, we compare the distributions of cells from a given cell type to a random sample, of the same size, from all cells in the experiment using the Mann-Whitney $U$ test for 10,000 iterations. We calculated the overall $\mathrm{p}$-value as the average number of times the observed distribution was significantly different than the permuted distribution.

We calculate 3 different metrics for each cell used with the described permutation test approach. Normalized Gene Expression use the Log10 Transcripts Per Million (TPM) UMI counts. Male-biased Enrichment Scores used Gene Set Enrichment Analysis (GSEA) with TPM UMI counts and L3 bulk testis-baised expressed genes to estimate an cell level enrichment score. Finally, we compared X and 4th expression with the major autosomal arms (2nd and 3rd) on an individual cell basis. We calculated the X:A,A, and 4,4:A,A ratios for each cell by taking the total X-linked (or 4th-linked) reads divided by the total autosomal-linked reads (2nd and 3rd) normalized by the number of genes per chromosome using all genes and the three subsets of genes with "housekeeping" characteristics.

We list all genes, strains, genetic reagents, antibodies, sequence based reagents, chemical compounds, commercial assays and kits, large-scale datasets, software and algorithms, and others used in our experiments in the FlyBase provided Author Reagent format (Supplementary Dataset 6).

Reporting summary. Further information on research design is available in the Nature Research Reporting Summary linked to this article.

\section{Data availability}

All raw sequencing data has been deposited and is publicly available through the NCBI Gene Expression Omnibus under accession codes "GSE125947”, "GSE115511", and "GSE115478"). Projections of the expression of each Drosophila gene onto the UMPA can be accessed at the NIH Figshare (https://doi.org/10.35092/yhjc.11950746). All data repository details and links are listed in the FlyBase Author Reagent format

(Supplementary Dataset 6). All other relevant data supporting the key findings of this study are available within the article and its Supplementary Information files or from the corresponding author upon reasonable request. Source data are provided with this paper in the Supplementary Data files.

\section{Code availability}

All code is at Zenodo (https://doi.org/10.5281/zenodo.3973174). All software packages and environments used are also listed in the FlyBase Author Reagent format (Supplementary Dataset 6).

Received: 11 April 2020; Accepted: 22 December 2020; Published online: 09 February 2021

\section{References}

1. Bachtrog, D. Y-chromosome evolution: emerging insights into processes of Ychromosome degeneration. Nat. Rev. Genet. 14, 113-124 (2013). 
2. Carvalho, A. B., Dobo, B. A., Vibranovski, M. D. \& Clark, A. G. Identification of five new genes on the Y chromosome of Drosophila melanogaster. Proc. Natl Acad. Sci. USA 98, 13225-13230 (2001).

3. Vicoso, B. \& Bachtrog, D. Reversal of an ancient sex chromosome to an autosome in Drosophila. Nature 499, 332-335 (2013).

4. Riddle, N. C. \& Elgin, S. C. R. The Drosophila dot chromosome: where genes flourish amidst repeats. Genetics 210, 757-772 (2018)

5. Vicoso, B. \& Charlesworth, B. Evolution on the X chromosome: unusual patterns and processes. Nat. Rev. Genet. 7, 645-653 (2006).

6. Khil, P. P., Smirnova, N. A., Romanienko, P. J. \& Camerini-Otero, R. D. The mouse $\mathrm{X}$ chromosome is enriched for sex-biased genes not subject to selection by meiotic sex chromosome inactivation. Nat. Genet. 36, 642-646 (2004).

7. Kelly, W. G. et al. X-chromosome silencing in the germline of C. elegans. Development 129, 479-492 (2002).

8. Parisi, M. et al. Paucity of genes on the Drosophila X chromosome showing male-biased expression. Science 299, 697-700 (2003).

9. Lee, J. T. Sex chromosome inactivation: the importance of pairing. Curr. Biol. 15, R249-R252 (2005).

10. Vibranovski, M. D. Meiotic sex chromosome inactivation in Drosophila. J. Genomics 2, 104-117 (2014).

11. Meiklejohn, C. D., Landeen, E. L., Cook, J. M., Kingan, S. B. \& Presgraves, D. C. Sex chromosome-specific regulation in the Drosophila male germline but little evidence for chromosomal dosage compensation or meiotic inactivation. PLoS Biol. 9, el001126 (2011).

12. Betrán, E., Thornton, K. \& Long, M. Retroposed new genes out of the $\mathrm{X}$ in Drosophila. Genome Res. 12, 1854-1859 (2002).

13. Sturgill, D., Zhang, Y., Parisi, M. \& Oliver, B. Demasculinization of X chromosomes in the Drosophila genus. Nature 450, 238-241 (2007).

14. Vibranovski, M. D., Zhang, Y. \& Long, M. General gene movement off the X chromosome in the Drosophila genus. Genome Res. 19, 897-903 (2009).

15. Turner, J. M. A. Meiotic silencing in mammals. Annu. Rev. Genet. 49, 395-412 (2015).

16. Huynh, K. D. \& Lee, J. T. X-chromosome inactivation: a hypothesis linking ontogeny and phylogeny. Nat. Rev. Genet. 6, 410-418 (2005).

17. Lu, L.-Y. \& Yu, X. Double-strand break repair on sex chromosomes: challenges during male meiotic prophase. Cell Cycle 14, 516-525 (2015).

18. Wu, C. I. \& Xu, E. Y. Sexual antagonism and X inactivation-the SAXI hypothesis. Trends Genet. 19, 243-247 (2003).

19. Handel, M. A. The XY body: a specialized meiotic chromatin domain. Exp. Cell Res. 296, 57-63 (2004).

20. McKee, B. D., Yan, R. \& Tsai, J.-H. Meiosis in male Drosophila. Spermatogenesis 2, 167-184 (2012).

21. Deng, X. et al. Evidence for compensatory upregulation of expressed X-linked genes in mammals, Caenorhabditis elegans and Drosophila melanogaster. Nat. Genet. 43, 1179-1185 (2011).

22. Kuroda, M. I., Hilfiker, A. \& Lucchesi, J. C. Dosage compensation in Drosophila-a Model for the coordinate regulation of transcription. Genetics 204, 435-450 (2016).

23. Rastelli, L. \& Kuroda, M. I. An analysis of maleless and histone H4 acetylation in Drosophila melanogaster spermatogenesis. Mech. Dev. 71, 107-117 (1998).

24. Oliver, B. Genetic control of germline sexual dimorphism in Drosophila. Int. Rev. Cytol. 219, 1-60 (2002).

25. Vibranovski, M. D., Lopes, H. F., Karr, T. L. \& Long, M. Stage-specific expression profiling of Drosophila spermatogenesis suggests that meiotic sex chromosome inactivation drives genomic relocation of testis-expressed genes. PLoS Genet. 5, e1000731 (2009).

26. Vibranovski, M. D. et al. Re-analysis of the larval testis data on meiotic sex chromosome inactivation revealed evidence for tissue-specific gene expression related to the drosophila X chromosome. BMC Biol. 10, 49 (2012). author reply 50.

27. Gupta, V. et al. Global analysis of X-chromosome dosage compensation. J. Biol. 5, 3 (2006)

28. Hense, W., Baines, J. F. \& Parsch, J. X chromosome inactivation during Drosophila spermatogenesis. PLoS Biol. 5, e273 (2007).

29. Rice, W. R. Sex chromosomes and the evolution of sexual dimorphism. Evolution 38, 735-742 (1984).

30. Hess, O. \& Meyer, G. F. Genetic activities of the Y chromosome in Drosophila during spermatogenesis. Adv. Genet. 14, 171-223 (1968).

31. Wagner, A., Regev, A. \& Yosef, N. Revealing the vectors of cellular identity with single-cell genomics. Nat. Biotechnol. 34, 1145-1160 (2016).

32. Hennig, W. Spermatogenesis in Drosophila. Int. J. Dev. Biol. 40, 167-176 (1996).

33. Fuller, M. T. Genetic control of cell proliferation and differentiation in Drosophila spermatogenesis. Semin. Cell Dev. Biol. 9, 433-444 (1998).

34. Greenspan, L. J., de Cuevas, M. \& Matunis, E. Genetics of gonadal stem cell renewal. Annu. Rev. Cell Dev. Biol. 31, 291-315 (2015).

35. Gönczy, P., Matunis, E. \& DiNardo, S. bag-of-marbles and benign gonial cell neoplasm act in the germline to restrict proliferation during Drosophila spermatogenesis. Development 124, 4361-4371 (1997).
36. Matunis, E., Tran, J., Gönczy, P., Caldwell, K. \& DiNardo, S. punt and schnurri regulate a somatically derived signal that restricts proliferation of committed progenitors in the germline. Development 124, 4383-4391 (1997).

37. Fairchild, M. J., Smendziuk, C. M. \& Tanentzapf, G. A somatic permeability barrier around the germline is essential for Drosophila spermatogenesis. Development 142, 268-281 (2015).

38. Nanda, S. et al. Sox100B, a Drosophila group E Sox-domain gene, is required for somatic testis differentiation. Sex. Dev. 3, 26-37 (2009).

39. Gvakharia, B. O., Kilgore, J. A., Bebas, P. \& Giebultowicz, J. M. Temporal and spatial expression of the period gene in the reproductive system of the codling moth. J. Biol. Rhythms 15, 4-12 (2000).

40. Bakken, T. E. et al. Single-nucleus and single-cell transcriptomes compared in matched cortical cell types. PLoS ONE 13, e0209648 (2018).

41. Lun, A. T. L. et al. EmptyDrops: distinguishing cells from empty droplets in droplet-based single-cell RNA sequencing data. Genome Biol. 20, 63 (2019).

42. Wolock, S. L., Lopez, R. \& Klein, A. M. Scrublet: computational identification of cell doublets in single-cell transcriptomic data. Cell Syst. 8, 281-291.e9 (2019).

43. Butler, A., Hoffman, P., Smibert, P., Papalexi, E. \& Satija, R. Integrating singlecell transcriptomic data across different conditions, technologies, and species. Nat. Biotechnol. 36, 411-420 (2018).

44. Kelso, R. J. et al. Flytrap, a database documenting a GFP protein-trap insertion screen in Drosophila melanogaster. Nucleic Acids Res. 32, D418-D420 (2004).

45. Kibanov, M. V. et al. A novel organelle, the piNG-body, in the nuage of Drosophila male germ cells is associated with piRNA-mediated gene silencing. Mol. Biol. Cell 22, 3410-3419 (2011).

46. Monk, A. C., Abud, H. E. \& Hime, G. R. Dmp53 is sequestered to nuclear bodies in spermatogonia of Drosophila melanogaster. Cell Tissue Res. 350, 385-394 (2012).

47. Kappes, F. et al. The DEK oncoprotein is a $\mathrm{Su}(\mathrm{var})$ that is essential to heterochromatin integrity. Genes Dev. 25, 673-678 (2011).

48. Chalkley, G. E. et al. The transcriptional coactivator SAYP is a trithorax group signature subunit of the PBAP chromatin remodeling complex. Mol. Cell. Biol. 28, 2920-2929 (2008).

49. Lu, C. \& Fuller, M. T. Recruitment of mediator complex by cell type and stagespecific factors required for tissue-specific TAF dependent gene activation in an adult stem cell lineage. PLoS Genet. 11, e1005701 (2015).

50. Leser, K., Awe, S., Barckmann, B., Renkawitz-Pohl, R. \& Rathke, C. The bromodomain-containing protein $\mathrm{tBRD}-1$ is specifically expressed in spermatocytes and is essential for male fertility. Biol. Open 1, 597-606 (2012).

51. Theofel, I. et al. tBRD-1 and tBRD-2 regulate expression of genes necessary for spermatid differentiation. Biol. Open 6, 439-448 (2017).

52. Chavez, J. et al. dAdd1 and dXNP prevent genome instability by maintaining HP1a localization at Drosophila telomeres. Chromosoma 126, 697-712 (2017).

53. Alekseyenko, A. A. et al. Heterochromatin-associated interactions of Drosophila HP1a with dADD1, HIPP1, and repetitive RNAs. Genes Dev. 28, 1445-1460 (2014).

54. White-Cooper, H. Spermatogenesis: analysis of meiosis and morphogenesis. Methods Mol. Biol. 247, 45-75 (2004).

55. Hempel, L. U., Rathke, C., Raja, S. J. \& Renkawitz-Pohl, R. In Drosophila, don juan and don juan like encode proteins of the spermatid nucleus and the flagellum and both are regulated at the transcriptional level by the TAF II80 cannonball while translational repression is achieved by distinct elements. Dev. Dyn. 235, 1053-1064 (2006).

56. Eberhart, C. G., Maines, J. Z. \& Wasserman, S. A. Meiotic cell cycle requirement for a fly homologue of human Deleted in Azoospermia. Nature 381, 783-785 (1996).

57. Maines, J. Z. \& Wasserman, S. A. Post-transcriptional regulation of the meiotic Cdc25 protein Twine by the Dazl orthologue Boule. Nat. Cell Biol. 1, 171-174 (1999).

58. Li, M. A., Alls, J. D., Avancini, R. M., Koo, K. \& Godt, D. The large Maf factor Traffic Jam controls gonad morphogenesis in Drosophila. Nat. Cell Biol. 5, 994-1000 (2003).

59. Hempel, L. U. \& Oliver, B. Sex-specific doublesexm expression in subsets of Drosophila somatic gonad cells. BMC Dev. Biol. 7, 113 (2007).

60. Caldwell, J. C., Fineberg, S. K. \& Eberl, D. F. reduced ocelli encodes the leucine rich repeat protein Pray For Elves in Drosophila melanogaster. Fly 1, 146-152 (2007).

61. Qu, Y. et al. Efa6 protects axons and regulates their growth and branching by inhibiting microtubule polymerisation at the cortex. Elife 8, e50319 (2019).

62. Xing, G. et al. Drosophila neuroligin3 regulates neuromuscular junction development and synaptic differentiation. J. Biol. Chem. 289, 31867-31877 (2014).

63. Kim, S. E., Coste, B., Chadha, A., Cook, B. \& Patapoutian, A. The role of Drosophila Piezo in mechanical nociception. Nature 483, 209-212 (2012).

64. Witt, E., Benjamin, S., Svetec, N. \& Zhao, L. Testis single-cell RNA-seq reveals the dynamics of de novo gene transcription and germline mutational bias in Drosophila. Elife 8, e47138 (2019). 
65. Wang, P. J., McCarrey, J. R., Yang, F. \& Page, D. C. An abundance of X-linked genes expressed in spermatogonia. Nat. Genet. 27, 422-426 (2001).

66. Redhouse, J. L., Mozziconacci, J. \& White, R. A. H. Co-transcriptional architecture in a Y loop in Drosophila melanogaster. Chromosoma 120, 399-407 (2011).

67. Fingerhut, J. M., Moran, J. V. \& Yamashita, Y. M. Satellite DNA-containing gigantic introns in a unique gene expression program during Drosophila spermatogenesis. PLoS Genet. 15, e1008028 (2019).

68. Ravasi, T. et al. An atlas of combinatorial transcriptional regulation in mouse and man. Cell 140, 744-752 (2010).

69. Yanai, I. et al. Genome-wide midrange transcription profiles reveal expression level relationships in human tissue specification. Bioinformatics 21, 650-659 (2005).

70. Cooper, K. W. Normal spermatogenesis in Drosophila. in Biology of Drosophila 1-61 (1965).

71. Thomas, S. E. \& McKee, B. D. Analysis of chromosome dynamics and chromosomal proteins in Drosophila spermatocytes. Methods Mol. Biol. 558, 217-234 (2009).

72. Tsai, J.-H., Yan, R. \& McKee, B. D. Homolog pairing and sister chromatid cohesion in heterochromatin in Drosophila male meiosis I. Chromosoma 120, 335-351 (2011).

73. Reugels, A. M., Kurek, R., Lammermann, U. \& Bünemann, H. Mega-introns in the dynein gene $\operatorname{DhDhc7}(Y)$ on the heterochromatic $\mathrm{Y}$ chromosome give rise to the giant threads loops in primary spermatocytes of Drosophila hydei. Genetics 154, 759-769 (2000).

74. Lifschytz, E. \& Lindsley, D. L. The role of X-chromosome inactivation during spermatogenesis (Drosophila-allocycly-chromosome evolution-male sterilitydosage compensation). Proc. Natl Acad. Sci. USA 69, 182-186 (1972).

75. Greil, F. \& Ahmad, K. Nucleolar dominance of the Y chromosome in Drosophila melanogaster. Genetics 191, 1119-1128 (2012).

76. Jowhar, Z. et al. Effects of human sex chromosome dosage on spatial chromosome organization. Mol. Biol. Cell 29, 2458-2469 (2018).

77. Phatnani, H. P. \& Greenleaf, A. L. Phosphorylation and functions of the RNA polymerase II CTD. Genes Dev. 20, 2922-2936 (2006).

78. El-Sharnouby, S., Redhouse, J. \& White, R. A. H. Genome-wide and cellspecific epigenetic analysis challenges the role of polycomb in Drosophila spermatogenesis. PLoS Genet. 9, e1003842 (2013).

79. Parsch, J., Meiklejohn, C. D., Hauschteck-Jungen, E., Hunziker, P. \& Hartl, D. L. Molecular evolution of the ocnus and janus genes in the Drosophila melanogaster species subgroup. Mol. Biol. Evol. 18, 801-811 (2001).

80. Carrel, L. \& Willard, H. F. X-inactivation profile reveals extensive variability in X-linked gene expression in females. Nature 434, 400-404 (2005)

81. Hsieh, T. \& Brutlag, D. Sequence and sequence variation within the $1.688 \mathrm{~g} / \mathrm{cm} 3$ satellite DNA of Drosophila melanogaster. J. Mol. Biol. 135, 465-481 (1979).

82. Pinkel, D., Straume, T. \& Gray, J. W. Cytogenetic analysis using quantitative, high-sensitivity, fluorescence hybridization. Proc. Natl. Acad. Sci. USA 83 2934-2938 (1986).

83. Cabral-de-Mello, D. C., Moura, R. C. \& Martins, C. Chromosomal mapping of repetitive DNAs in the beetle Dichotomius geminatus provides the first evidence for an association of $5 \mathrm{~S}$ rRNA and histone $\mathrm{H} 3$ genes in insects, and repetitive DNA similarity between the B chromosome and A complement. Heredity 104, 393-400 (2010).

84. Lee, H. et al. Effects of gene dose, chromatin, and network topology on expression in Drosophila melanogaster. PLoS Genet. 12, e1006295 (2016).

85. Martin, M. Cutadapt removes adapter sequences from high-throughput sequencing reads. EMBnet. J. 17, 10-12 (2011).

86. Kim, D., Langmead, B. \& Salzberg, S. L. HISAT: a fast spliced aligner with low memory requirements. Nat. Methods 12, 357-360 (2015).

87. Li, H. et al. The sequence alignment/map format and SAMtools. Bioinformatics 25, 2078-2079 (2009)

88. Liao, Y., Smyth, G. K. \& Shi, W. The Subread aligner: fast, accurate and scalable read mapping by seed-and-vote. Nucleic Acids Res. 41, e108 (2013).

89. Wingett, S. W. \& Andrews, S. FastQ Screen: a tool for multi-genome mapping and quality control. F1000Res. 7, 1338 (2018).

90. Liao, Y., Smyth, G. K. \& Shi, W. featureCounts: an efficient general purpose program for assigning sequence reads to genomic features. Bioinformatics $\mathbf{3 0}$ 923-930 (2014).

91. Yang, H. et al. Re-annotation of eight Drosophila genomes. Life Sci. Alliance 1, e201800156 (2018)
92. Stuart, T. et al. Comprehensive integration of single-cell data. Cell 177, 1888-1902.e21 (2019).

93. Hicks, S. C., Townes, F. W., Teng, M. \& Irizarry, R. A. Missing data and technical variability in single-cell RNA-sequencing experiments. Biostatistics 19, 562-578 (2018).

\section{Acknowledgements}

This work utilized the computational resources of the NIH HPC Biowulf cluster (http:// hpc.nih.gov), NCBI and FlyBase databases, and the Bloomington Drosophila Stock Center. We thank Bob Johnston for oligopaint probe libraries, Gustavo Kuhn for providing the $\mathrm{X}$ chromosome satellite probe, Eduardo Gorab for total RNA Pol-II antibodies, and Veronic Souza for checking the 4th chromosome localization in our experiments. This research was supported in part by the Intramural Research Program of the NIH, The National Institute of Diabetes and Digestive and Kidney Diseases (DK015600) and the National Heart Lung and Blood Institute (HL006126), the National Institute of General Medical Sciences (GM126752 and GM120107), the Eunice Kennedy Shriver National Institute of Child Health and Human Development (HD052937) the São Paulo Research Foundation (JP 2015/20844-4, CEPID 2013/08028-1, PT 2015/16661-1, MS 2017/26609-2, MS 2017/14923-4, DD 2019/15212-0 and DD 2019/14788-5).

\section{Author contributions}

S.M., J.M.F., B.O., M.D.V. and E.M. conceptualized the project; S.M. and J.M.F. developed single cell methodology; J.M.F. developed code; M.A., M.M.L.S.P., C.C.A., D.C.C M., K.C., Z.D., K.M., C.A.M., O.M.P.G., E.R., M.S. and K.Y. validated results; J.M.F., M.A., B.J.G., C.C.A., and C.A.M. performed formal analysis; S.M., M.A., B.J.G., M.M.L.S P., C.C.A., D.C.C.M., K.C., S.D., Z.D., K.M., C.A.M., O.M.P.G., E.R., M.S., K.Y., H.Y., H.E.S. and V.S. performed experiments; J.M.F. curated metadata; S.M., J.M.F., B.O., M.D.V. and E.M. wrote the original draft; S.M., J.M.F., M.A., B.O., M.D.V. and E.M. edited; S.M., J.M.F., M.A., C.C.A., B.O. and M.D.V. prepared figures; S.M., H.E.S., V.S., N.R., B.O., M.D.V. and E.M. directed the work; S.M. and B.O. administered the project; N.M.R., B.O., M.D.V., M.A. and E.M. acquired funding.

\section{Competing interests}

The authors declare no competing interests.

\section{Additional information}

Supplementary information is available for this paper at https://doi.org/10.1038/s41467 021-20897-y.

Correspondence and requests for materials should be addressed to B.O.

Peer review information Nature Communications thanks Todd Nystul and the other, anonymous, reviewer(s) for their contribution to the peer review of this work. Peer reviewer reports are available.

Reprints and permission information is available at http://www.nature.com/reprints

Publisher's note Springer Nature remains neutral with regard to jurisdictional claims in published maps and institutional affiliations.

\begin{abstract}
(c) (i) Open Access This article is licensed under a Creative Commons BY Attribution 4.0 International License, which permits use, sharing, adaptation, distribution and reproduction in any medium or format, as long as you give appropriate credit to the original author(s) and the source, provide a link to the Creative Commons license, and indicate if changes were made. The images or other third party material in this article are included in the article's Creative Commons license, unless indicated otherwise in a credit line to the material. If material is not included in the article's Creative Commons license and your intended use is not permitted by statutory regulation or exceeds the permitted use, you will need to obtain permission directly from the copyright holder. To view a copy of this license, visit http://creativecommons.org/ licenses/by/4.0/.
\end{abstract}

This is a U.S. government work and not under copyright protection in the U.S.; foreign copyright protection may apply 2021 